\title{
Patient-relevant outcomes: what are we talking about? A scoping review to improve conceptual clarity
}

\author{
Christine Kersting $^{1 *} \mathbb{D}$, Malte Kneer ${ }^{1}$ and Anne Barzel ${ }^{1,2}$
}

\begin{abstract}
Background: With respect to patient-centered care, measuring care effects based on patient-relevant outcomes is becoming increasingly important. There is some uncertainty about what outcomes are particularly relevant to patients and who determines their relevance. To determine this, we conducted a scoping review of the international literature with the aim to improve the conceptual clarity regarding (1) the terminology used for supposedly patient-relevant outcomes, (2) the variety of outcomes considered patient-relevant, and (3) justifications for the choice of these specific outcomes.
\end{abstract}

Methods: We conducted a systematic search in Embase, PubMed (including Medline), Cochrane Central, Scopus, and Google Scholar with a special focus on article titles. Search terms included patient-relevant, patient-important, patient-preferred, and outcome(s), endpoint(s), parameter(s), indicator(s). We limited the search period from January 2000 to July 2019. Full-text articles reporting outcomes that were described as patient-relevant met the inclusion criteria. Two researchers independently analyzed all eligible articles applying quantitative and structuring content analysis.

Results: We identified 155 articles, 44 of which met the inclusion criteria. A content analysis revealed 35 different terms used with regard to patient-relevant outcomes. However, authors predominantly referred to patientimportant outcomes (23 articles, 52.3\%) and patient-relevant outcomes (17 articles, 38.6\%). A structuring content analysis of all extracted outcomes revealed a total of 281 codes, pooled in 32 inductive categories. Among these, the following categories dominated: symptoms, adverse events/complications, survival/mortality, pain. In just 16 of the articles (36.4\%), authors provided justifications for the choice of the outcome being based either on patient and/or expert opinions. In another 13 articles (29.5\%), no justification was provided.

Conclusion: This scoping review on patient-relevant outcomes was driven by the questions (1) what outcomes are particularly relevant to patients, and (2) who determines their relevance. We found a wide range of supposedly patientrelevant outcomes, with only one third of articles involving patients in the justification of the outcome selection. In view of this conceptual uncertainty it appears difficult to determine or even to compare a particular patient benefit of interventions. A set of generic outcomes relevant to patients would be helpful to contribute to a consistent understanding of patient relevance.

Keywords: Patient-relevant outcome, Patient relevance, Patient preference, Patient-centered care, Patient involvement

\footnotetext{
* Correspondence: Christine.Kersting@uni-wh.de

'Institute of General Practice and Interprofessional Care, Faculty of Health/

School of Medicine, Witten/Herdecke University, Alfred-Herrhausen-Str. 50, 58448 Witten, Germany

Full list of author information is available at the end of the article
}

(c) The Author(s). 2020 Open Access This article is licensed under a Creative Commons Attribution 4.0 International License, which permits use, sharing, adaptation, distribution and reproduction in any medium or format, as long as you give appropriate credit to the original author(s) and the source, provide a link to the Creative Commons licence, and indicate if changes were made. The images or other third party material in this article are included in the article's Creative Commons licence, unless indicated otherwise in a credit line to the material. If material is not included in the article's Creative Commons licence and your intended use is not permitted by statutory regulation or exceeds the permitted use, you will need to obtain permission directly from the copyright holder. To view a copy of this licence, visit http://creativecommons.org/licenses/by/4.0/ The Creative Commons Public Domain Dedication waiver (http://creativecommons.org/publicdomain/zero/1.0/) applies to the data made available in this article, unless otherwise stated in a credit line to the data. 


\section{Background}

Patient-centered care aims to place individuals, their values, preferences, life and health goals at the heart of the care process and to actively involve patients in care decisions [1]. In the sense of shared decision-making, involving patients in decisions implies that patients are adequately informed about existing care options and their potential effects, understand these options, and are given the opportunity to explore what is most relevant to them in order to make a choice based on their personal preferences [2]. In our understanding, this means that for shared decision-making, effect measurements based on parameters that matter to patients are urgently needed. This assumes that studies examine the effects of care that is based on outcomes which are relevant to patients, but so far systematic reviews conclude that such patientrelevant outcomes are underrepresented in recent clinical trials [3-5]. Indeed, this raises the question as to what outcomes are relevant to patients and who determines their relevance.

In Germany, the Institute for Quality and Efficiency in Health Care (IQEHC) officially examines the benefits and harms of medical interventions for patients. To this end, the IQEHC considers parameters to be relevant to patients when they represent how a patient feels, functions or survives; notably mortality, morbidity, and quality of life [6]. However, this understanding of patient relevance is based on a definition of the Biomarkers Definition Working Group on clinical endpoints, which does not contain any information about patient relevance [7]. Similarly to the IQEHC's understanding of patient-relevant outcomes, the working group on quality of care and patient safety research of the German Network for Health Services Research (DNVF) mentions survival and quality of life as factors that are relevant to patients, but also includes social aspects, such as social reintegration, in its interpretation [8]. Generally, these outcomes are based on the understanding that patientrelevant outcomes reflect the effects of changes in the individual patient's health status [8].

However, neither the definition provided by the IQEHC nor that provided by the working group of the DNVF offered us a comprehensive explanation as to why the outcomes mentioned are considered to be relevant to patients. Shifting the view from the German to the international context, we aimed to examine which understanding of patient relevance and which outcomes are common to international research and thereby hoped to improve the conceptual clarity of patient relevance. To this end, we conducted a scoping review of the international literature with regard to the following research questions: (1) What terminology is used for supposedly patient-relevant outcomes? (2) What outcomes are considered to be relevant to patients? (3)
What explanations are provided to justify the relevance of these specific outcomes for patients?

\section{Methods}

We report our scoping review in accordance with the PRISMA extension for scoping reviews (PRISMA-ScR) [9].

\section{Data sources and search strategy}

We conducted a systematic literature search in Embase, PubMed (including Medline), Cochrane Central, Scopus, and Google Scholar. Our search strategy considered German and English references published between January 1st, 2000 and July 31st, 2019. Restricting the search period to the past 20 years was considered reasonable to concentrate on more recent research. In order to identify references that clearly focus on the topic of interest, we additionally restricted the search to the article titles. Titles had to include at least one of the following terms: (patient-relevant OR patient-important OR patientpreferred) AND (outcome(s) OR endpoint(s) OR parame$\operatorname{ter}(s) \mathrm{OR}$ indicator(s)). The full search strategies for each database are outlined in Additional file 1.

\section{Study selection}

After deleting all duplicates, two of the researchers (Christine Kersting (CK), Malte Kneer (MK)) reviewed all records retrieved from the database search: In the first step, we independently screened the records to limit the search results to English- and German-language articles with full-text availability published in journals. This was reasonable as we aimed to examine whether there is, in fact, a common and consistent understanding of patient relevance in international research. Accordingly, we excluded doctoral theses if not officially published, conference abstracts, commentaries on previously published articles, opinions, debates, or editorials, records on other topics, and those without full-text availability. In the second step, we independently reviewed the full texts of the remaining articles to check whether they met the inclusion criteria for our review. We defined articles as eligible when they reported outcomes that were described by the authors as relevant to patients. In case of disagreements we discussed these until a consensus was achieved.

\section{Data extraction and analysis}

In line with the three research questions of this scoping review, we analyzed all eligible articles regarding (1) the terminology used, (2) the outcomes described as patientrelevant, and (3) the justifications for why these outcomes were considered to be of relevance to patients. For the analysis we used both quantitative content analysis and qualitative structuring content analysis [10]. 


\section{Terminology}

All terms that were used to describe patient-relevant outcomes were assessed and extracted in original spelling to collect synonyms; however, paraphrases such as "outcomes that matter to patients" were not considered synonyms. Finally, we applied frequency calculations to quantify the number of different terms used per article and the overall number of different terms identified.

\section{Outcomes}

In a second step all outcomes described as relevant to patients were extracted from the articles and structured for the underlying issues (thematically) using inductive categories. The code set was extended continuously as new categories emerged during the full-text analyses. We additionally examined whether specific outcome categories were used more often than others by calculating frequencies and percentages.

\section{Justification}

We analyzed whether the articles provided a justification as to why the outcomes described were considered to be relevant to patients. These justifications were categorized inductively. Based on this, we distributed the outcome categories that were identified in the previous step to the justifications provided.

In a subgroup analysis we focused on articles that actively involved patients or experts in the definition of patient-relevant outcomes. We examined whether the outcomes defined in these articles were valid for specific patient groups only and whether these outcomes were different from those described as relevant to patients in articles that did not involve patients or experts. Additionally, we stratified for studies involving patients, studies involving experts, and those involving both, and compared the outcome categories between these groups.

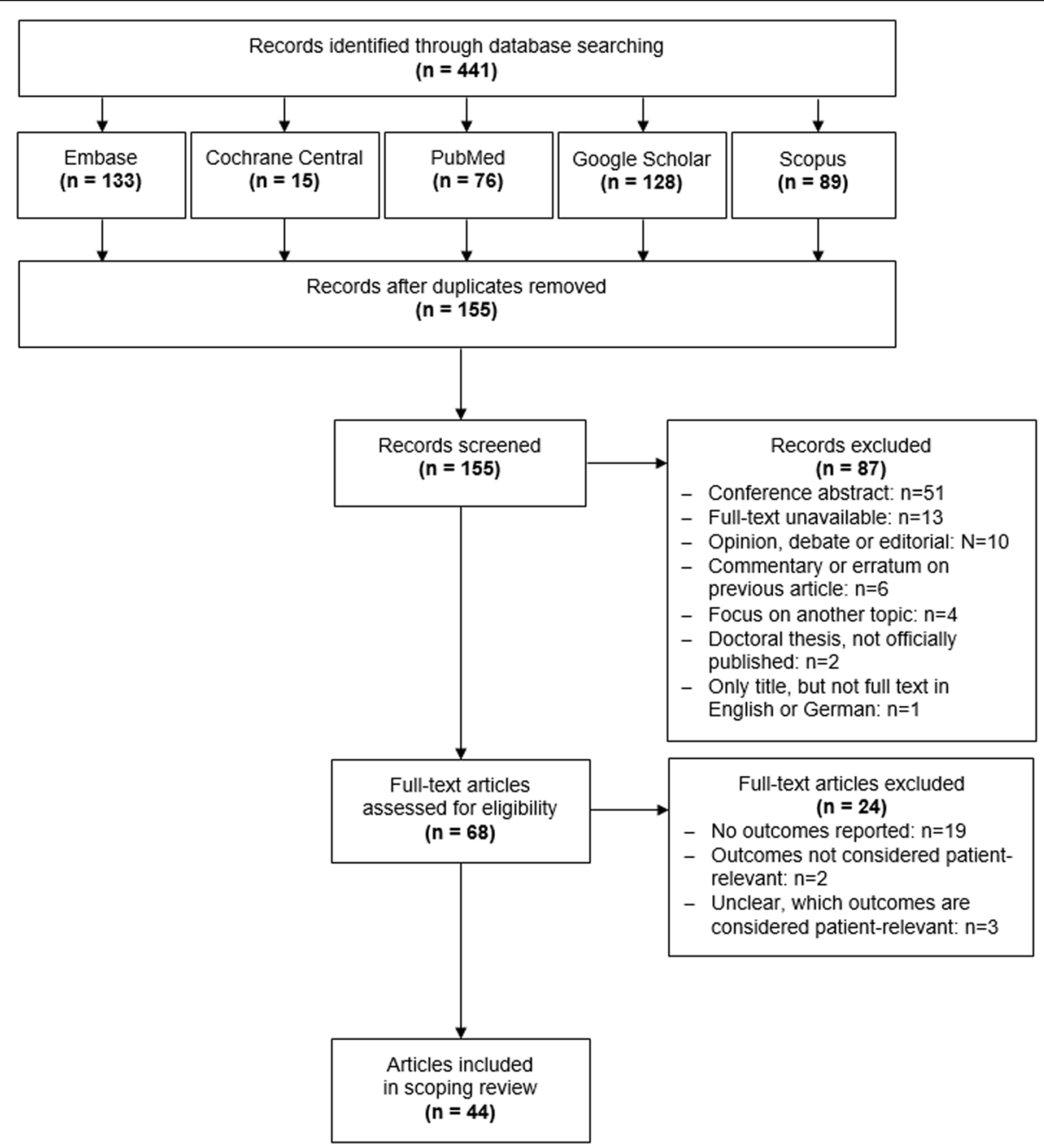

Fig. 1 Flow chart depicting study selection 
Table 1 Characteristics of the 16 articles involving patients and/or experts to justify patient relevance of outcomes

\begin{tabular}{|c|c|c|c|c|c|}
\hline Authors & Objective/ motivation of article & Type of article/ study & $\begin{array}{l}\text { Terms used } \\
\text { synonymously }\end{array}$ & $\begin{array}{l}\text { Indicator disease/ } \\
\text { intervention }\end{array}$ & $\begin{array}{l}\text { Outcomes considered patient- } \\
\text { relevant [instrument, if } \\
\text { applicable] }\end{array}$ \\
\hline $\begin{array}{l}\text { Blome } \\
\text { et al. } 2009 \\
\text { [11] }\end{array}$ & $\begin{array}{l}\text { Development and validation of a } \\
\text { specific version of the German patient } \\
\text { benefit index (PBI-S) to be used in the } \\
\text { treatment of pruritus (PBI-P) }\end{array}$ & $\begin{array}{l}\text { Mixed methods study } \\
\text { involving patients and } \\
\text { experts }\end{array}$ & $\begin{array}{l}5 \text { terms: } \\
\text { - patient- } \\
\text { relevant } \\
\text { benefit } \\
\text { - patient- } \\
\text { relevant } \\
\text { treatment } \\
\text { benefit } \\
\text { - patient } \\
\text { preference } \\
\text { - patient- } \\
\text { reported } \\
\text { benefit } \\
\text { - patient- } \\
\text { relevant } \\
\text { outcome }\end{array}$ & Pruritus & $\begin{array}{l}\text { In decreasing order of } \\
\text { importance, i.a.: } \\
\text { - no longer experience itching } \\
\text { - find a clear diagnosis and } \\
\text { therapy } \\
\text { - have confidence in therapy } \\
\text { - be free of pain } \\
\text { - no longer have a burning } \\
\text { sensation on the skin } \\
\text { - be able to sleep better } \\
\text { - be less dependent on doctor } \\
\text { and clinic visits }\end{array}$ \\
\hline $\begin{array}{l}\text { Blome } \\
\text { et al. } 2014 \\
\text { [12] }\end{array}$ & $\begin{array}{l}\text { Development and validation of a } \\
\text { specific version of the German patient } \\
\text { benefit index (PBI-S) to be used in the } \\
\text { treatment of lymphedema and } \\
\text { lipedema (PBI-L) }\end{array}$ & $\begin{array}{l}\text { Mixed methods study } \\
\text { involving patients and } \\
\text { experts }\end{array}$ & $\begin{array}{l}3 \text { terms: } \\
\text { - patient- } \\
\text { relevant } \\
\text { outcome } \\
\text { - patient- } \\
\text { relevant } \\
\text { benefit } \\
\text { - patient- } \\
\text { relevant } \\
\text { treatment } \\
\text { benefit }\end{array}$ & $\begin{array}{l}\text { Lymphedema, } \\
\text { lipedema }\end{array}$ & $\begin{array}{l}\text { In decreasing order of } \\
\text { importance, i.a.: } \\
\text { - experience less swelling and } \\
\text { tension } \\
\text { - be free of pain } \\
\text { - avoid complications } \\
\text { - receive optimal hosiery (e.g., } \\
\text { color, fit, prescription quantity) } \\
\text { - be less restricted in your } \\
\text { ability to move around } \\
\text { - have no fear that the disease } \\
\text { will become worse } \\
\text { - find a clear diagnosis and } \\
\text { therapy }\end{array}$ \\
\hline $\begin{array}{l}\text { Cho et al. } \\
2019 \text { [13] }\end{array}$ & $\begin{array}{l}\text { Identification of patients'and } \\
\text { caregivers'priorities for outcomes in trials } \\
\text { on autosomal dominant polycystic } \\
\text { kidney disease }\end{array}$ & $\begin{array}{l}\text { Mixed methods study } \\
\text { involving patients } \\
\text { only }\end{array}$ & $\begin{array}{l}4 \text { terms: } \\
\text { - patient- } \\
\text { important } \\
\text { outcome } \\
\text { - patient- } \\
\text { prioritised } \\
\text { outcome } \\
\text { - patient- } \\
\text { centered } \\
\text { outcome } \\
\text { - patient- } \\
\text { reported } \\
\text { outcome }\end{array}$ & $\begin{array}{l}\text { Autosomal } \\
\text { dominant } \\
\text { polycystic kidney } \\
\text { disease }\end{array}$ & $\begin{array}{l}\text { In decreasing order of } \\
\text { importance, i.a.: } \\
\text { - kidney function } \\
\text { - end-stage kidney disease } \\
\text { - survival } \\
\text { - cyst size/growth } \\
\text { - cyst pain/bleeding } \\
\text { - blood pressure } \\
\text { - ability to work } \\
\text { - cerebral aneurysm/stroke } \\
\text { - mobility/physical function } \\
\text { - fatigue }\end{array}$ \\
\hline \multirow[t]{3}{*}{$\begin{array}{l}\text { Daeter } \\
\text { et al. } 2018 \\
\text { [14] }\end{array}$} & \multirow{3}{*}{$\begin{array}{l}\text { 1. Share the standard set of outcomes } \\
\text { for coronary artery disease developed by } \\
\text { Meetbaar Beter } \\
\text { 2. Illustrate how the standard set is } \\
\text { presented and published } \\
\text { Note: Meetbaar Beter aims to improve } \\
\text { the quality of cardiovascular care in } \\
\text { hospitals in the Netherlands by creating } \\
\text { transparency on patient-relevant out- } \\
\text { comes (Benchmarking) }\end{array}$} & \multirow[t]{3}{*}{$\begin{array}{l}\text { Delphi approach } \\
\text { among experts only }\end{array}$} & \multirow[t]{3}{*}{$\begin{array}{l}1 \text { term: } \\
\text { - patient- } \\
\text { relevant } \\
\text { outcome }\end{array}$} & $\begin{array}{l}\text { Coronary artery } \\
\text { disease (overall) }\end{array}$ & $\begin{array}{l}\text { - long-term survival ( } \leq 5 \text { years) } \\
\text { - 1-year mortality } \\
\text { - quality of life [SF-36] } \\
\text { - myocardial infarction ( } \leq 30 \\
\text { days) }\end{array}$ \\
\hline & & & & $\begin{array}{l}\text { Coronary artery } \\
\text { bypass grafting }\end{array}$ & $\begin{array}{l}\text { - } 120 \text {-day mortality } \\
\text { - surgical reexploration }(\leq 30 \\
\text { days) } \\
\text { - cerebrovascular accident ( } \leq 72 \\
\text { h) } \\
\text { - Deep sternal wound infection } \\
\text { ( } \leq 30 \text { days) } \\
\text { - free of myocardial infarction } \\
\text { - free of coronary artery } \\
\text { reintervention }\end{array}$ \\
\hline & & & & $\begin{array}{l}\text { Percutaneous } \\
\text { coronary } \\
\text { intervention }\end{array}$ & $\begin{array}{l}\text { - } 30 \text {-day mortality } \\
\text { - urgent coronary artery bypass } \\
\text { grafting ( } \leq 24 \mathrm{~h}) \\
\text { - occurrence of target vessel } \\
\text { revascularization ( } \leq 1 \text { year) }\end{array}$ \\
\hline
\end{tabular}


Table 1 Characteristics of the 16 articles involving patients and/or experts to justify patient relevance of outcomes (Continued)

\begin{tabular}{|c|c|c|c|c|c|}
\hline Authors & Objective/ motivation of article & Type of article/ study & $\begin{array}{l}\text { Terms used } \\
\text { synonymously }\end{array}$ & $\begin{array}{l}\text { Indicator disease/ } \\
\text { intervention }\end{array}$ & $\begin{array}{l}\text { Outcomes considered patient- } \\
\text { relevant [instrument, if } \\
\text { applicable] }\end{array}$ \\
\hline & & & & $\begin{array}{l}\text { Conservative } \\
\text { treatment }\end{array}$ & $\begin{array}{l}\text { - chest pain ( } \leq 1 \text { year) } \\
\text { - free of major adverse cardiac } \\
\text { event }\end{array}$ \\
\hline $\begin{array}{l}\text { Dinglas } \\
\text { et al. } 2018 \\
\text { [15] }\end{array}$ & $\begin{array}{l}\text { Synthesis of the literature with regard to } \\
\text { patient-important outcome for Intensive } \\
\text { Care Unit survivors focusing on a re- } \\
\text { search program on acute respiratory } \\
\text { failure }\end{array}$ & $\begin{array}{l}\text { Synthesis, Delphi } \\
\text { approach among } \\
\text { patients only }\end{array}$ & $\begin{array}{l}1 \text { term: } \\
\text { - patient- } \\
\text { important } \\
\text { outcome }\end{array}$ & $\begin{array}{l}\text { Acute respiratory } \\
\text { failure }\end{array}$ & $\begin{array}{l}\text { - survival } \\
\text { - physical function } \\
\text { - muscle and/or nerve function } \\
\text { - pulmonary function } \\
\text { - cognition } \\
\text { - mental health [HADS, IES-R] } \\
\text { - pain [EQ-5D pain question] } \\
\text { - health-related quality of life } \\
\text { [EQ-5D, SF-36] }\end{array}$ \\
\hline $\begin{array}{l}\text { Eiring et al. } \\
2016 \text { [16] }\end{array}$ & $\begin{array}{l}\text { 1. Investigate the relative importance of } \\
\text { patient-important outcomes in bipolar } \\
\text { disorder } \\
\text { 2. Construct a holistic taxonomy of } \\
\text { patient-important outcomes }\end{array}$ & $\begin{array}{l}\text { Mixed methods study } \\
\text { involving patients } \\
\text { only }\end{array}$ & $\begin{array}{l}2 \text { terms: } \\
\text { - patient- } \\
\text { important } \\
\text { outcome } \\
\text { - patient- } \\
\text { relevant } \\
\text { outcome }\end{array}$ & Bipolar disorder & $\begin{array}{l}\text { In decreasing order of } \\
\text { importance, i.a.: } \\
\text { - avoid serve depression } \\
\text { - avoid serve mania } \\
\text { - increase quality of life } \\
\text { - better functioning (school/ } \\
\text { work) } \\
\text { - better social functioning }\end{array}$ \\
\hline $\begin{array}{l}\text { Kinter } \\
\text { et al. } 2009 \\
{[17]}\end{array}$ & $\begin{array}{l}\text { 1. Identification of endpoints directly } \\
\text { from patients with schizophrenia } \\
\text { 2. Evaluation whether patients can } \\
\text { express which endpoints matter to } \\
\text { them } \\
\text { 3. Ranking of the relevant endpoints }\end{array}$ & $\begin{array}{l}\text { Mixed methods study } \\
\text { involving patients } \\
\text { only }\end{array}$ & $\begin{array}{l}5 \text { terms: } \\
\text { - patient- } \\
\text { relevant } \\
\text { endpoint } \\
\text { - patient- } \\
\text { relevant } \\
\text { benefit } \\
\text { - patient } \\
\text { endpoint } \\
\text { - patient } \\
\text { preference } \\
\text { - patient- } \\
\text { centered } \\
\text { endpoint }\end{array}$ & Schizophrenia & $\begin{array}{l}\text { In decreasing order of } \\
\text { importance, i.a.: } \\
\text { - clear thinking } \\
\text { - minimization of symptoms } \\
\text { - social activities } \\
\text { - daily activities } \\
\text { - supportive physician }\end{array}$ \\
\hline $\begin{array}{l}\text { Konkle } \\
\text { et al. } 2019 \\
{[18]}\end{array}$ & $\begin{array}{l}\text { Review of strengths and limitations of } \\
\text { outcome measures used in hemophilia } \\
\text { trials from a provider and patient } \\
\text { perspective }\end{array}$ & $\begin{array}{l}\text { Review involving } \\
\text { patients and experts }\end{array}$ & $\begin{array}{l}3 \text { terms: } \\
\text { - patient- } \\
\text { important } \\
\text { outcome } \\
\text { - patient- } \\
\text { relevant } \\
\text { outcome } \\
\text { - patient- } \\
\text { important } \\
\text { clinical } \\
\text { outcome }\end{array}$ & Hemophilia & $\begin{array}{l}\text { - frequency of bleeds } \\
\text { - factor activity level } \\
\text { - duration of expression } \\
\text { - chronic pain } \\
\text { - healthcare resource use } \\
\text { - mental health }\end{array}$ \\
\hline $\begin{array}{l}\text { Lindsley } \\
\text { et al. } 2018 \\
{[19]}\end{array}$ & $\begin{array}{l}\text { Identification and prioritization of clinical } \\
\text { questions and patient-relevant out- } \\
\text { comes for research associated with the } \\
\text { treatment of age-related macular } \\
\text { degeneration }\end{array}$ & $\begin{array}{l}\text { Delphi approach } \\
\text { among patients and } \\
\text { experts }\end{array}$ & $\begin{array}{l}1 \text { term: } \\
\text { - patient- } \\
\text { important } \\
\text { outcome }\end{array}$ & $\begin{array}{l}\text { Age-related } \\
\text { macular } \\
\text { degeneration }\end{array}$ & $\begin{array}{l}\text { Highly important to patients: } \\
\text { - choroidal neovascularization } \\
\text { - development of advanced } \\
\text { age-related macular } \\
\text { degeneration } \\
\text { - retinal hemorrhage } \\
\text { - gain of vision } \\
\text { - slowing vision loss } \\
\text { - serious ocular events }\end{array}$ \\
\hline $\begin{array}{l}\text { Murad } \\
\text { et al. } 2011 \\
\text { [20] }\end{array}$ & $\begin{array}{l}\text { Assessment of preferences of patients } \\
\text { with diabetes on how clinical trials } \\
\text { should be conducted with regard to } \\
\text { study design (pragmatic versus } \\
\text { explanatory) and endpoints (surrogate } \\
\text { versus patient-relevant) }\end{array}$ & $\begin{array}{l}\text { Cross-sectional study } \\
\text { involving patients } \\
\text { only }\end{array}$ & $\begin{array}{l}2 \text { terms: } \\
\text { - patient- } \\
\text { important } \\
\text { outcome } \\
\text { - patient } \\
\text { preference }\end{array}$ & Diabetes mellitus & $\begin{array}{l}\text { In decreasing order of } \\
\text { importance, i.a.: } \\
\text { - end-stage renal disease } \\
\text { - stroke } \\
\text { - myocardial infarction } \\
\text { - blindness } \\
\text { - HbA1c control } \\
\text { - death }\end{array}$ \\
\hline
\end{tabular}


Table 1 Characteristics of the 16 articles involving patients and/or experts to justify patient relevance of outcomes (Continued)

\begin{tabular}{|c|c|c|c|c|c|}
\hline Authors & Objective/ motivation of article & Type of article/ study & $\begin{array}{l}\text { Terms used } \\
\text { synonymously }\end{array}$ & $\begin{array}{l}\text { Indicator disease/ } \\
\text { intervention }\end{array}$ & $\begin{array}{l}\text { Outcomes considered patient- } \\
\text { relevant [instrument, if } \\
\text { applicable] }\end{array}$ \\
\hline $\begin{array}{l}\text { Nabbout } \\
\text { et al. } 2018 \\
\text { [21] }\end{array}$ & $\begin{array}{l}\text { Identification of a core set of patient- } \\
\text { and caregiver-relevant concepts to be } \\
\text { included in future clinical trials on dravet } \\
\text { syndrome }\end{array}$ & $\begin{array}{l}\text { Qualitative study, } \\
\text { Delphi approach } \\
\text { among caregivers and } \\
\text { experts }\end{array}$ & $\begin{array}{l}2 \text { terms: } \\
\text { - patient- and } \\
\text { caregiver- } \\
\text { relevant } \\
\text { outcome } \\
\text { - patient- and } \\
\text { caregiver- } \\
\text { relevant } \\
\text { endpoint }\end{array}$ & Dravet syndrome & $\begin{array}{l}\text { - seizures } \\
\text { - expressive communication of } \\
\text { the child } \\
\text { - receptive communication of } \\
\text { the child } \\
\text { - daily activities of the caregiver } \\
\text { - social functioning of the } \\
\text { caregiver }\end{array}$ \\
\hline $\begin{array}{l}\text { Sanderson } \\
\text { et al. } 2010 \\
\text { [22] }\end{array}$ & $\begin{array}{l}\text { Identification of treatment outcomes } \\
\text { important to patients with rheumatoid } \\
\text { arthritis receiving anti-tumor necrosis } \\
\text { factor therapy }\end{array}$ & $\begin{array}{l}\text { Qualitative study } \\
\text { involving patients } \\
\text { only }\end{array}$ & $\begin{array}{l}6 \text { terms: } \\
\text { - patient } \\
\text { outcome } \\
\text { - patient } \\
\text { priority } \\
\text { treatment } \\
\text { outcome } \\
\text { - patient } \\
\text { priority } \\
\text { outcome } \\
\text { - patients' } \\
\text { important } \\
\text { treatment } \\
\text { outcome } \\
\text { - patients' } \\
\text { important } \\
\text { outcome } \\
\text { - patient- } \\
\text { important } \\
\text { outcome }\end{array}$ & $\begin{array}{l}\text { Rheumatoid } \\
\text { arthritis }\end{array}$ & $\begin{array}{l}\text { - rheumatoid arthritis under } \\
\text { control (symptoms less, } \\
\text { rheumatoid arthritis stable, } \\
\text { medication effects) } \\
\text { - doing things (doing things, } \\
\text { able to plan) } \\
\text { - emotional health (positive } \\
\text { feelings, holistic identity, } \\
\text { positive mental changes, } \\
\text { better life) } \\
\text { - coping with illness (coping } \\
\text { with rheumatoid arthritis, } \\
\text { coping with health system) } \\
\text { - global outcomes (feeling well, } \\
\text { return to a normal life, feeling } \\
\text { (more) normal) }\end{array}$ \\
\hline $\begin{array}{l}\text { Sung et al. } \\
2014 \text { [23] }\end{array}$ & $\begin{array}{l}\text { Development of a comprehensive } \\
\text { conceptual framework representing the } \\
\text { relevant dimensions and outcomes } \\
\text { important to women with pelvic organ } \\
\text { prolapse }\end{array}$ & $\begin{array}{l}\text { Mixed methods study } \\
\text { involving patients } \\
\text { only }\end{array}$ & $\begin{array}{l}1 \text { term: } \\
\text { - patient- } \\
\text { important } \\
\text { outcome }\end{array}$ & $\begin{array}{l}\text { Pelvic organ } \\
\text { prolapse }\end{array}$ & $\begin{array}{l}\text { In decreasing order of } \\
\text { importance, i.a.: } \\
\text { - alleviation of physical bulge } \\
\text { symptoms and associated } \\
\text { discomfort } \\
\text { - improvement in physical } \\
\text { function } \\
\text { - improvement in sexual } \\
\text { function } \\
\text { - improvement in body image } \\
\text { perception } \\
\text { - improvement in social } \\
\text { function }\end{array}$ \\
\hline $\begin{array}{l}\text { Van der } \\
\text { Elst et al. } \\
2016 \text { [24] }\end{array}$ & $\begin{array}{l}\text { Better comprehension of the } \\
\text { perspective of patients with early } \\
\text { rheumatoid arthritis on preferred health } \\
\text { and treatment outcomes }\end{array}$ & $\begin{array}{l}\text { Qualitative study } \\
\text { involving patients } \\
\text { only }\end{array}$ & $\begin{array}{l}2 \text { terms: } \\
\text { - patient- } \\
\text { preferred } \\
\text { outcome } \\
\text { - patient- } \\
\text { preferred } \\
\text { health and } \\
\text { treatment } \\
\text { outcome }\end{array}$ & $\begin{array}{l}\text { Early rheumatoid } \\
\text { arthritis }\end{array}$ & $\begin{array}{l}\text { - aspects of disease control, e.g., } \\
\text { prevention or control of joint } \\
\text { damage, less medication } \\
\text { - physical aspects, e.g., relief of } \\
\text { pain and other physical } \\
\text { symptoms, improved joint } \\
\text { function and mobility } \\
\text { - aspects of participation, e.g., } \\
\text { performing activities of daily } \\
\text { living, engaging in work and/ } \\
\text { or leisure } \\
\text { - mental aspects, e.g., emotional } \\
\text { well-being, life enjoyment }\end{array}$ \\
\hline $\begin{array}{l}\text { Wilson } \\
\text { et al. } 2019 \\
{[25]}\end{array}$ & $\begin{array}{l}\text { Synthesis of evidence in all outcome } \\
\text { domains identified as important by } \\
\text { patients undergoing unicompartmental } \\
\text { or total knee replacement and outcome } \\
\text { domains commonly used in other } \\
\text { studies }\end{array}$ & $\begin{array}{l}\text { Systematic review \& } \\
\text { meta-analysis involv- } \\
\text { ing patients and refer- } \\
\text { ring to other studies }\end{array}$ & $\begin{array}{l}1 \text { term: } \\
\text { - patient } \\
\text { relevant } \\
\text { outcome }\end{array}$ & $\begin{array}{l}\text { Unicompartmental } \\
\text { and total knee } \\
\text { replacement in } \\
\text { osteoarthritis }\end{array}$ & $\begin{array}{l}\text { - hospital admission impact: } \\
\text { length of operation, length of } \\
\text { hospital stay } \\
\text { - risk of early complications } \\
\text { (myocardial infarction, stroke, } \\
\text { venous thromboembolism, } \\
\text { deep infection) or early } \\
\text { mortality } \\
\text { - success of operation: range of }\end{array}$ \\
\hline
\end{tabular}


Table 1 Characteristics of the 16 articles involving patients and/or experts to justify patient relevance of outcomes (Continued)

\begin{tabular}{|c|c|c|c|c|c|}
\hline Authors & Objective/ motivation of article & Type of article/ study & $\begin{array}{l}\text { Terms used } \\
\text { synonymously }\end{array}$ & $\begin{array}{l}\text { Indicator disease/ } \\
\text { intervention }\end{array}$ & $\begin{array}{l}\text { Outcomes considered patient- } \\
\text { relevant [instrument, if } \\
\text { applicable] }\end{array}$ \\
\hline \multirow{6}{*}{$\begin{array}{l}\text { Van } \\
\text { Veghel } \\
\text { et al. } 2016 \\
\text { [26] }\end{array}$} & \multirow{6}{*}{$\begin{array}{l}\text { Presentation and discussion of the } \\
\text { patient-relevant outcomes of Meetbaar } \\
\text { Beter for coronary artery disease and } \\
\text { aortic valve disease, focusing on the sur- } \\
\text { gical procedures coronary artery bypass } \\
\text { grafting, percutaneous coronary inter- } \\
\text { vention, aortic valve replacement and } \\
\text { transcatheter aortic valve implantation }\end{array}$} & \multirow{6}{*}{$\begin{array}{l}\text { Database analysis } \\
\text { involving experts only }\end{array}$} & \multirow{6}{*}{$\begin{array}{l}3 \text { terms: } \\
\text { - patient- } \\
\text { relevant } \\
\text { outcome } \\
\text { - patient- } \\
\text { relevant } \\
\text { health } \\
\text { outcome } \\
\text { - patient- } \\
\text { oriented } \\
\text { outcome }\end{array}$} & & $\begin{array}{l}\text { movement achieved or } \\
\text { kneeling ability, reduction in } \\
\text { pain, improvement in function } \\
\text { - reoperation or revision rate } \\
\text { - rate of recovery: rate of return } \\
\text { to work, rate of return to } \\
\text { sporting activities }\end{array}$ \\
\hline & & & & $\begin{array}{l}\text { Coronary artery } \\
\text { disease (overall) }\end{array}$ & $\begin{array}{l}\text { - readmission due to myocardia } \\
\text { infarction ( } \leq 30 \text { days) }\end{array}$ \\
\hline & & & & $\begin{array}{l}\text { Coronary artery } \\
\text { bypass grafting }\end{array}$ & $\begin{array}{l}\text { - 120-day mortality } \\
\text { - quality of life [SF-36] }\end{array}$ \\
\hline & & & & $\begin{array}{l}\text { Percutaneous } \\
\text { coronary } \\
\text { intervention }\end{array}$ & $\begin{array}{l}\text { - 1-year mortality } \\
\text { - Occurrence of target vessel } \\
\text { revascularization ( } \leq 1 \text { year) }\end{array}$ \\
\hline & & & & $\begin{array}{l}\text { Aortic valve } \\
\text { replacement }\end{array}$ & $\begin{array}{l}\text { - 120-day mortality } \\
\text { - long-term survival }\end{array}$ \\
\hline & & & & $\begin{array}{l}\text { Transcatheter } \\
\text { aortic valve } \\
\text { implantation }\end{array}$ & $\begin{array}{l}\text { - 120-day mortality } \\
\text { - implantation of a new } \\
\text { permanent pacemaker }(\leq 30 \\
\text { days) }\end{array}$ \\
\hline
\end{tabular}

\section{Results}

\section{Literature search}

Excluding duplicates, the literature search yielded 155 records (Fig. 1). During the first screening, we excluded 87 records because the full-text papers were unavailable, not written in English or German, focused on another topic, or were conference abstracts, commentaries on previously published articles, opinions, debates, or editorials. Full-text screening of the remaining 68 articles resulted in 44 articles fulfilling the inclusion criteria for this review. Details on all studies are provided in Tables 1, 2, 3 and 4 .

Regarding the study design, we found a mixture of methodological approaches consisting mainly of reviews (16 of 44, 36.4\%) [3-5, 18, 25, 27-29, 31-36, 40, 48] and qualitative or mixed-methods studies including Delphi approaches (12 of 44, 27.3\%) [11-17, 19, 21-24]. Whereas the reviews considered studies across different countries, other studies related to a specific country were conducted mainly in Sweden $(n=9)[30,37-39,43$, $45-47,51]$ and Germany $(n=4)[11,12,49,50]$.

\section{Terminology}

Based on the 44 articles included in this review, we identified 35 different terms for patient-relevant outcomes (Table 5). Of these 35 terms, the two most frequently used terms were patient-important outcome (identified in 23 articles, 52.3\%) and patient-relevant outcome (identified in 17 articles, $38.6 \%$ ).

In 21 articles $(47.7 \%)$ one term was used consistently for supposedly patient-relevant outcomes; in 14 articles
(31.8\%) two terms were used, and in the remaining nine articles (20.5\%) up to six different terms were used to describe the outcomes. We were not able to identify different patterns in terminology across countries. The terms identified per article are demonstrated in Tables $1,2,3$ and 4 .

\section{Outcomes}

A structuring content analysis of the outcomes extracted from the 44 articles resulted in 281 codes, from which 32 inductive categories were composed. Irrespective of the different shadings per bar, Fig. 2 illustrates these 32 categories in ascending order of frequency (. The most strongly represented categories were: symptoms $(n=34$ codes, 12.1\%), adverse events/complications $(n=31$ codes, $11.0 \%)$, survival/mortality ( $n=31$ codes, $11.0 \%$ ), pain $(n=26$ codes, $9.3 \%)$, generic quality of life/health status ( $n=24$ codes, $8.5 \%$ ), (co)morbidity/secondary diseases ( $n=24$ codes, $8.5 \%$ ), and physical function/functional status $(n=20$ codes, $7.1 \%)$. The outcomes extracted per article are listed in Tables 1, 2, 3 and 4.

\section{Justification}

In about one third ( $n=13,29.5 \%)$ of the 44 articles analyzed, no reason was provided why the outcomes reported were considered to be relevant to patients (Table 4). However, in other studies we found different explanations justifying the selection of patient-relevant outcomes that refer mainly to three core issues: 
Table 2 Characteristics of the 12 articles referring to other studies or special classifications to justify patient relevance of outcomes

\begin{tabular}{|c|c|c|c|c|c|}
\hline Authors & Objective/ motivation of article & $\begin{array}{l}\text { Type of article/ } \\
\text { study }\end{array}$ & $\begin{array}{l}\text { Terms used } \\
\text { synonymously }\end{array}$ & $\begin{array}{l}\text { Indicator } \\
\text { disease/ } \\
\text { intervention }\end{array}$ & $\begin{array}{l}\text { Outcomes considered patient- } \\
\text { relevant [instrument, if applicable] }\end{array}$ \\
\hline $\begin{array}{l}\text { Adie et al. } \\
2017 \text { [4] }\end{array}$ & $\begin{array}{l}\text { Determination of the proportion of patient- } \\
\text { important primary outcomes in surgical ran- } \\
\text { domized controlled trials }\end{array}$ & $\begin{array}{l}\text { Systematic } \\
\text { review \& meta- } \\
\text { analysis }\end{array}$ & $\begin{array}{l}2 \text { terms: } \\
\text { - patient- } \\
\text { important } \\
\text { outcome } \\
\text { - patient- } \\
\text { centered } \\
\text { outcome }\end{array}$ & $\begin{array}{l}\text { Surgical } \\
\text { interventions }\end{array}$ & $\begin{array}{l}\text { - mortality/survival } \\
\text { - pain } \\
\text { - function } \\
\text { - quality of life } \\
\text { - any morbid event or symptom } \\
\text { - patient satisfaction } \\
\text { - any intervention to address the } \\
\text { previous outcomes }\end{array}$ \\
\hline $\begin{array}{l}\text { Ameur et al. } \\
2017 \text { [27] }\end{array}$ & $\begin{array}{l}\text { Determination whether recently published } \\
\text { an ongoing systematic reviews with meta- } \\
\text { analyses of therapeutic interventions assess } \\
\text { patient-important outcomes }\end{array}$ & $\begin{array}{l}\text { Methodological } \\
\text { review }\end{array}$ & $\begin{array}{l}1 \text { term: } \\
\text { - patient- } \\
\text { important } \\
\text { outcome }\end{array}$ & $\begin{array}{l}\text { Generic; } \\
\text { therapeutic } \\
\text { interventions }\end{array}$ & $\begin{array}{l}\text { - mortality } \\
\text { - clinical events } \\
\text { - pain } \\
\text { - quality of life } \\
\text { - therapeutic decision } \\
\text { - function }\end{array}$ \\
\hline $\begin{array}{l}\text { Fei et al. } \\
2018 \text { [28] }\end{array}$ & $\begin{array}{l}\text { Examination of the impact of adding } \\
\text { ezetimibe to statins on patient-important } \\
\text { outcomes in patients at high cardiovascular } \\
\text { risk }\end{array}$ & $\begin{array}{l}\text { Narrative } \\
\text { systematic } \\
\text { review }\end{array}$ & $\begin{array}{l}1 \text { term: } \\
\text { - patient- } \\
\text { important } \\
\text { outcome }\end{array}$ & $\begin{array}{l}\text { High } \\
\text { cardiovascular } \\
\text { risk }\end{array}$ & $\begin{array}{l}\text { - all-cause mortality } \\
\text { - cardiovascular mortality } \\
\text { - non-fatal stroke } \\
\text { - non-fatal myocardial infarction } \\
\text { - adverse events }\end{array}$ \\
\hline $\begin{array}{l}\text { Gaudry et al. } \\
2017 \text { [5] }\end{array}$ & $\begin{array}{l}\text { Investigation whether randomized } \\
\text { controlled trials in critically ill patients assess } \\
\text { patient-important outcomes }\end{array}$ & $\begin{array}{l}\text { Systematic } \\
\text { review }\end{array}$ & $\begin{array}{l}1 \text { term: } \\
\text { - patient- } \\
\text { important } \\
\text { outcome }\end{array}$ & Critical illness & $\begin{array}{l}\text { - mortality } \\
\text { - quality of life after Intensive Care } \\
\text { Unit discharge } \\
\text { - functional, cognitive, and } \\
\text { neurological outcomes after } \\
\text { Intensive Care Unit discharge }\end{array}$ \\
\hline $\begin{array}{l}\text { Kvitkina } \\
\text { et al. } 2014 \\
\text { [29] }\end{array}$ & $\begin{array}{l}\text { Description of the feasibility of the early } \\
\text { benefit assessment of novel agents on the } \\
\text { basis of patient-relevant outcomes by char- } \\
\text { acterizing the outcomes available in the }\end{array}$ & $\begin{array}{l}\text { Systematic } \\
\text { Review }\end{array}$ & $\begin{array}{l}1 \text { term: } \\
\text { - patient- } \\
\text { relevant } \\
\text { outcome }\end{array}$ & $\begin{array}{l}\text { Drugs (novel } \\
\text { agents) }\end{array}$ & $\begin{array}{l}\text { - mortality } \\
\text { - morbidity } \\
\text { - health-related quality of life } \\
\text { - adverse events }\end{array}$ \\
\hline
\end{tabular}
companies dossiers and comparing them to outcomes defined as patient-relevant by the German Institute for Quality and Efficiency in Health Care

Roos et al. Evaluation of patient-relevant outcomes 2000 [30] preoperatively and three months after partial meniscectomy

$\begin{array}{ll}\text { Prospective } & 1 \text { term: } \\ \text { follow-up study } & \begin{array}{l}\text { - patient- } \\ \text { relevant } \\ \text { outcome }\end{array}\end{array}$

Schumacher Assessment of current approaches to et al. 2016 measure the impact of tuberculosis nucleic [31] acid amplification tests on patientimportant outcomes in adults with possibly tuberculosis and/or drug-resistant tuberculosis

Singh et al. Simulation of the long-term effect of novel 2017 [32] agents versus chemotherapy-based regimens on progression-free survival, overall survival and health-related quality of life in patients with chronic lymphocytic leukemia

Wieseler et al. 2013
Determination of the information gain between clinical study reports and publicly available sources for patient-relevant outcomes included in health technology assessments for drugs

\begin{tabular}{|c|c|c|c|}
\hline $\begin{array}{l}\text { Methodological } \\
\text { review }\end{array}$ & $\begin{array}{l}2 \text { terms: } \\
\text { - patient- } \\
\text { important } \\
\text { outcome } \\
\text { - patient } \\
\text { outcome }\end{array}$ & $\begin{array}{l}\text { Tuberculosis } \\
\text { nucleic acid } \\
\text { amplification } \\
\text { tests }\end{array}$ & $\begin{array}{l}\text { - culture conversion } \\
\text { - tuberculosis treatment outcomes } \\
\text { - infection control/ contact tracing } \\
\text { - morbidity } \\
\text { - mortality }\end{array}$ \\
\hline $\begin{array}{l}\text { Review \& data } \\
\text { simulation }\end{array}$ & $\begin{array}{l}2 \text { terms: } \\
\text { - patient- } \\
\text { relevant } \\
\text { outcome } \\
\text { - patient- } \\
\text { relevant } \\
\text { benefit }\end{array}$ & $\begin{array}{l}\text { Chronic } \\
\text { lymphocytic } \\
\text { leukemia }\end{array}$ & $\begin{array}{l}\text { - progression-free survival } \\
\text { - overall survival } \\
\text { - quality-adjusted life years } \\
\text { - post-progression survival }\end{array}$ \\
\hline $\begin{array}{l}\text { Systematic } \\
\text { review }\end{array}$ & $\begin{array}{l}2 \text { terms: } \\
\text { - patient- } \\
\text { relevant clin- } \\
\text { ical trial } \\
\text { outcome } \\
\text { - patient- } \\
\text { relevant } \\
\text { outcome }\end{array}$ & Drugs & $\begin{array}{l}\text { - mortality } \\
\text { - clinical events } \\
\text { - symptoms } \\
\text { - health-related quality of life } \\
\text { - (serious) adverse events }\end{array}$ \\
\hline
\end{tabular}

outcome

Partial $\quad$ - general health status [SF-36] meniscectomy - knee-specific health status: pain, symptoms, activities of daily life, sports and recreation function, knee-related quality of life, functional disability [KOOS, Lysholm Knee Score]

culture conversion

- infection control/ contact tracing

morbidity

outcome

outcome

outcome 
Table 2 Characteristics of the 12 articles referring to other studies or special classifications to justify patient relevance of outcomes (Continued)

\begin{tabular}{|c|c|c|c|c|c|}
\hline Authors & Objective/ motivation of article & $\begin{array}{l}\text { Type of article/ } \\
\text { study }\end{array}$ & $\begin{array}{l}\text { Terms used } \\
\text { synonymously }\end{array}$ & $\begin{array}{l}\text { Indicator } \\
\text { disease/ } \\
\text { intervention }\end{array}$ & $\begin{array}{l}\text { Outcomes considered patient- } \\
\text { relevant [instrument, if applicable] }\end{array}$ \\
\hline $\begin{array}{l}\text { Yordanov } \\
\text { et al. } 2018 \\
\text { [34] }\end{array}$ & $\begin{array}{l}\text { 1. Evaluation whether the outcomes } \\
\text { reported in the summary of finding table of } \\
\text { Cochrane reviews could be considered } \\
\text { patient-important } \\
\text { 2. Evaluation of the quality of evidence for } \\
\text { these outcomes }\end{array}$ & $\begin{array}{l}\text { Methodological } \\
\text { review }\end{array}$ & $\begin{array}{l}1 \text { term } \\
\text { - patient- } \\
\text { important } \\
\text { outcome / } \\
\text { PIO }\end{array}$ & Generic & $\begin{array}{l}\text { - mortality } \\
\text { - other clinical events (e.g., } \\
\text { myocardial infarction, stroke) } \\
\text { - adverse events } \\
\text { - function (e.g., anxiety, depression, } \\
\text { disability) } \\
\text { - pain } \\
\text { - quality of life } \\
\text { - therapeutic decisions }\end{array}$ \\
\hline $\begin{array}{l}\text { El Dib et al. } \\
2017 \text { [35] }\end{array}$ & $\begin{array}{l}\text { Review of randomized controlled trials on } \\
\text { diagnostic tests with regard to their topic } \\
\text { areas, population, setting, study groups, } \\
\text { patient-important outcomes, risk of bias, } \\
\text { and results }\end{array}$ & $\begin{array}{l}\text { Systematic } \\
\text { review }\end{array}$ & $\begin{array}{l}1 \text { term: } \\
\text { - patient- } \\
\text { important } \\
\text { outcome }\end{array}$ & $\begin{array}{l}\text { Diagnostic } \\
\text { tests }\end{array}$ & $\begin{array}{l}\text { - mortality } \\
\text { - morbidity } \\
\text { - symptoms } \\
\text { - quality of life } \\
\text { - functional status }\end{array}$ \\
\hline $\begin{array}{l}\text { Fayed et al. } \\
2014 \text { [36] }\end{array}$ & $\begin{array}{l}\text { 1. Review to which extent activity and } \\
\text { participation outcomes are included in } \\
\text { pediatric clinical trials } \\
\text { 2. Determination what characteristics are } \\
\text { associated with using theses outcomes }\end{array}$ & $\begin{array}{l}\text { Systematic } \\
\text { review }\end{array}$ & $\begin{array}{l}3 \text { terms: } \\
\text { - patient- } \\
\text { important } \\
\text { outcome } \\
\text { - patient- } \\
\text { important ac- } \\
\text { tivity and par- } \\
\text { ticipation } \\
\text { outcome } \\
\text { - child and } \\
\text { family- } \\
\text { important } \\
\text { outcome }\end{array}$ & $\begin{array}{l}\text { Generic; } \\
\text { children with } \\
\text { chronic } \\
\text { conditions }\end{array}$ & $\begin{array}{l}\text { - body function } \\
\text { - activity } \\
\text { - participation } \\
\text { - environmental factors } \\
\text { - personal factors } \\
\text { - health condition } \\
\text { - general health }\end{array}$ \\
\hline
\end{tabular}

1. Involvement of patients and/or experts was apparent in 16 out of 44 articles (36.4\%) (Table 1). To explain their choice of outcomes authors referred either to patients only $(n=9)[13,15-17,20,22-25]$ or to experts only $(n=2)[14,26]$ or to both patients' and experts' opinion $(n=5)[11,12,18,19,21]$. One study on early childhood disease included caregivers instead of patients [21], another one on critical disease included caregivers in addition to patients [13]. Sample sizes differed largely from smaller qualitative studies including 10 to 30 patients recruited in the health care setting [21, 22, 24] to a cross-sectional study in a random sample of about 2000 individuals invited by mail [20].

2. Referral to other studies $[4,5,27-34]$ or special classification $[35,36]$ was used as another source of supposedly patient-relevant outcomes (Table 2). These articles $(n=12,27.3 \%)$ referred their choice of outcomes mainly to reviews $[4,5,27,28,34]$ or to a specific classification such as the International Classification of Functioning, Disability and Health [36].

3. A few articles considered patient-reported outcomes equivalent to patient-reported outcomes $(n=3,6.8 \%$; Table 3$)$. In one of these articles authors argued that patient-relevant outcomes rely exclusively on the information provided by patients themselves [39].

The different shading of the bars in Fig. 2 illustrates the allocation of the outcomes extracted from the studies considering justifications of patient relevance provided by the authors. It shows that more popular outcome categories were justified on the basis of different explanations, whereas more seldom categories and those based on social aspects were commonly traced back to patients and/or experts.

\section{Subgroup analysis of articles involving patients and/or experts}

All articles that had actively involved patients and/or experts focused on a specific disease (Table 1), but outcomes considered patient-relevant showed a widespread distribution with regard to the 32 inductive categories identified, referring to as many as 29 different categories. However, these studies did not include outcomes related to satisfaction, self-efficacy, or disease-specific quality of life/health status (Fig. 2). Interestingly, we found that 12 of the 32 inductive categories were only used in articles with patient and/or expert involvement such as physiological functioning, ability to fulfil social functions, 
Table 3 Characteristics of the 3 articles equating patient-relevant outcomes with self-reported outcomes

\begin{tabular}{|c|c|c|c|c|c|}
\hline Authors & Objective/ motivation of article & $\begin{array}{l}\text { Type of article/ } \\
\text { study }\end{array}$ & $\begin{array}{l}\text { Terms used } \\
\text { synonymously }\end{array}$ & $\begin{array}{l}\text { Indicator disease/ } \\
\text { intervention }\end{array}$ & $\begin{array}{l}\text { Outcomes considered patient- } \\
\text { relevant [instrument, if applicable] }\end{array}$ \\
\hline $\begin{array}{l}\text { Nilsdotter } \\
\text { et al. } 2009 \\
\text { [37] }\end{array}$ & $\begin{array}{l}\text { 1. Description of outcomes up to five } \\
\text { years after total knee replacement for } \\
\text { osteoarthritis from the patients' } \\
\text { perspective } \\
\text { 2. Evaluation to what extent patients } \\
\text { performed physical activities after } \\
\text { total knee replacement } \\
\text { 3. Identification of preoperative } \\
\text { characteristics predicting } \\
\text { postoperative outcomes }\end{array}$ & $\begin{array}{l}\text { Prospective } \\
\text { follow-up study }\end{array}$ & $\begin{array}{l}2 \text { terms: } \\
\text { - patient- } \\
\text { relevant } \\
\text { outcome } \\
\text { - self-reported } \\
\text { outcome }\end{array}$ & $\begin{array}{l}\text { Total knee } \\
\text { replacement in } \\
\text { osteoarthritis }\end{array}$ & $\begin{array}{l}\text { - general health status [SF-36] } \\
\text { - knee-specific health status: pain, } \\
\text { stiffness, physical function } \\
\text { [WOMAC] } \\
\text { - general comorbidity }\end{array}$ \\
\hline $\begin{array}{l}\text { Nilsdotter, } \\
\text { Isaksson } \\
2010 \text { [38] }\end{array}$ & $\begin{array}{l}\text { Prospective evaluation of patient- } \\
\text { relevant outcomes seven years after } \\
\text { total hip replacement for osteoarth- } \\
\text { ritis focusing on pain and physical } \\
\text { function }\end{array}$ & $\begin{array}{l}\text { Prospective } \\
\text { cohort study with } \\
\text { matched controls } \\
\text { without hip } \\
\text { complaints }\end{array}$ & $\begin{array}{l}2 \text { terms: } \\
\text { - patient- } \\
\text { relevant } \\
\text { outcome } \\
\text { - patient- } \\
\text { reported } \\
\text { outcome }\end{array}$ & $\begin{array}{l}\text { Hybrid total hip } \\
\text { replacement and } \\
\text { demented total hip } \\
\text { replacement for } \\
\text { osteoarthritis }\end{array}$ & $\begin{array}{l}\text { - general health status [SF-36] } \\
\text { - knee-specific health status: pain, } \\
\text { stiffness, physical function } \\
\text { [WOMAC] } \\
\text { - postoperative complications } \\
\text { - general comorbidity } \\
\text { - musculoskeletal comorbidity: need } \\
\text { of walking assistance, walking } \\
\text { distance, pain, need for analgesics, } \\
\text { joint replacement in contralateral } \\
\text { hip or in knee, fractures } \\
\text { - patient satisfaction }\end{array}$ \\
\hline $\begin{array}{l}\text { Nilsdotter, } \\
\text { Lohmander } \\
2003 \text { [39] }\end{array}$ & $\begin{array}{l}\text { Investigation of pre- and } \\
\text { postoperative patient-relevant out- } \\
\text { comes between hybrid total hip re- } \\
\text { placement and cemented total hip } \\
\text { replacement in patients with } \\
\text { osteoarthritis }\end{array}$ & $\begin{array}{l}\text { Prospective } \\
\text { cohort study }\end{array}$ & $\begin{array}{l}2 \text { terms: } \\
\text { - patient- } \\
\text { relevant } \\
\text { outcome } \\
\text { - patient- } \\
\text { relevant } \\
\text { measure }\end{array}$ & $\begin{array}{l}\text { Total hip } \\
\text { replacement for } \\
\text { osteoarthritis }\end{array}$ & $\begin{array}{l}\text { - general health status [SF-36] } \\
\text { - knee-specific health status: pain, } \\
\text { stiffness, physical function } \\
\text { [WOMAC] } \\
\text { - postoperative complications } \\
\text { - general comorbidity } \\
\text { - musculoskeletal comorbidity: need } \\
\text { of walking assistance, walking } \\
\text { distance, pain, need for analgesics, } \\
\text { joint replacement in contralateral } \\
\text { hip or in knee, fractures }\end{array}$ \\
\hline
\end{tabular}

independency, reliability of diagnosis and therapy, and confidence in therapy. However, outcomes most commonly described as patient-relevant, such as symptoms, adverse events/complications, survival/mortality, and (co)morbidity/secondary disease were identical to those most commonly described as patient-relevant in the other 28 articles not involving patients and/or experts (Fig. 2).

Stratifying articles involving patients and/or experts $(n=16)$ by the manner of involvement showed that sur$\mathrm{vival} /$ mortality was mentioned especially in studies involving experts only. On the other hand, outcomes identified only in studies involving patients and/or experts (i.e. physiological functioning, reliability of diagnosis and therapy, or confidence in therapy) were traced back to those studies involving either patients only or both patients and experts.

\section{Discussion}

In this scoping review, we analyzed the international literature of the last 20 years with regard to patient relevance. We found a large variety of terms used as well as numerous supposedly patient-relevant outcomes. No more than one third of the articles referred to the patients', the caregivers' or the experts' opinions to explain the relevance of the outcomes reported. All in all, we were not able to identify a consistent concept or understanding of patient-relevant outcomes. Table 6 summarizes the main findings and implications for future research.

According to our analysis, the reasons for the identified ambiguity appear to be heterogeneous. Some articles did not differentiate between patient-reported and patient-relevant outcomes [37-39]. Other articles emphasized a potential overlap of the terms, but noted that not every outcome which can be reported by patients necessarily has to be relevant for them $[16,18,23,24$, 41]. Two articles on chronically ill children expanded the terminology from patients to the family to underline the social context and impact [21,36]. In both studies, the target group was involved in the definition of relevant outcomes. Indeed, such patient- or target groupdriven approaches are not common practice yet: two thirds of the articles we analyzed referred to previous 
Table 4 Characteristics of the 13 studies not providing any justification for the patient relevance of outcomes

\begin{tabular}{|c|c|c|c|c|c|}
\hline Authors & Objective/ motivation of article & $\begin{array}{l}\text { Type of } \\
\text { article/ study }\end{array}$ & $\begin{array}{l}\text { Terms used } \\
\text { synonymously }\end{array}$ & $\begin{array}{l}\text { Indicator } \\
\text { disease/ } \\
\text { intervention }\end{array}$ & $\begin{array}{l}\text { Outcomes considered patient- } \\
\text { relevant [instrument, if applicable] }\end{array}$ \\
\hline $\begin{array}{l}\text { Agarwal } \\
\text { et al. } 2017 \\
\text { [40] }\end{array}$ & $\begin{array}{l}\text { Examination to what extent Cochrane } \\
\text { and non-Cochrane reviews report abso- } \\
\text { lute effects for patient-important out- } \\
\text { comes in the abstract }\end{array}$ & $\begin{array}{l}\text { Systematic } \\
\text { review }\end{array}$ & $\begin{array}{l}1 \text { term: } \\
\text { - patient-important } \\
\text { outcome }\end{array}$ & Generic & $\begin{array}{l}\text { - mortality } \\
\text { - morbidity } \\
\text { - symptoms } \\
\text { - quality of life } \\
\text { - functional status }\end{array}$ \\
\hline $\begin{array}{l}\text { Cao et al. } \\
2014[41]\end{array}$ & $\begin{array}{l}\text { Comparison of the efficacy of two } \\
\text { commonly used Chinese patent } \\
\text { medicines for patients with angina } \\
\text { pectoris }\end{array}$ & $\begin{array}{l}\text { Study } \\
\text { protocol for a } \\
\text { randomized } \\
\text { controlled } \\
\text { trial }\end{array}$ & $\begin{array}{l}1 \text { term: } \\
\text { - patient-important } \\
\text { outcome }\end{array}$ & $\begin{array}{l}\text { Angina } \\
\text { pectoris }\end{array}$ & $\begin{array}{l}\text { - short of breath } \\
\text { - fatigue } \\
\text { - palpitations } \\
\text { - sweating }\end{array}$ \\
\hline $\begin{array}{l}\text { Cleveringa } \\
\text { et al. } 2010 \\
\text { [42] }\end{array}$ & $\begin{array}{l}\text { Determination of the effects of the } \\
\text { Diabetes Care Protocol on patient- } \\
\text { important outcomes } \\
\text { Note: When applying the Diabetes Care } \\
\text { Protocol, routine diabetes care is } \\
\text { delegated to a nurse, who uses a } \\
\text { computerized decision support system } \\
\text { to structure diabetes care and set } \\
\text { targets }\end{array}$ & $\begin{array}{l}\text { Cluster- } \\
\text { randomized } \\
\text { trial }\end{array}$ & $\begin{array}{l}1 \text { term: } \\
\text { - patient-important } \\
\text { outcome }\end{array}$ & $\begin{array}{l}\text { Type } 2 \\
\text { diabetes }\end{array}$ & $\begin{array}{l}\text { - diabetes-specific health status: psy- } \\
\text { chological distress, barriers to ac- } \\
\text { tivity, disinhibited eating [DHP-18] } \\
\text { - general health status [SF-36, EQ- } \\
\text { 5D] } \\
\text { - treatment satisfaction [DTSQ- } \\
\text { status] } \\
\text { - self-efficacy [DES-SF] }\end{array}$ \\
\hline $\begin{array}{l}\text { Englund } \\
\text { et al. } 2001 \\
\text { [43] }\end{array}$ & $\begin{array}{l}\text { Evaluation of long-term patient- } \\
\text { relevant outcomes after removal of } \\
\text { knee meniscus }\end{array}$ & $\begin{array}{l}\text { Retrospective } \\
\text { cohort study }\end{array}$ & $\begin{array}{l}2 \text { terms: } \\
\text { - patient-relevant } \\
\text { outcome } \\
\text { - self-administered out- } \\
\text { come measure }\end{array}$ & Meniscectomy & $\begin{array}{l}\text { - general health status [SF-36] } \\
\text { - knee-specific health status: pain, } \\
\text { symptoms, activities of daily life, } \\
\text { sports and recreation function, } \\
\text { knee-related quality of life [KOOS] }\end{array}$ \\
\hline $\begin{array}{l}\text { Gandhi } \\
\text { et al. } 2008 \\
\text { [3] }\end{array}$ & $\begin{array}{l}\text { Determination to what extent } \\
\text { registered randomized controlled trials } \\
\text { among patients with diabetes plan to } \\
\text { assess patient-important outcomes }\end{array}$ & $\begin{array}{l}\text { Systematic } \\
\text { review }\end{array}$ & $\begin{array}{l}1 \text { term: } \\
\text { - patient-important } \\
\text { outcome }\end{array}$ & Diabetes & $\begin{array}{l}\text { - mortality } \\
\text { - quality of life } \\
\text { - major morbid events } \\
\text { - minor morbid events } \\
\text { - pain } \\
\text { - functional status }\end{array}$ \\
\hline $\begin{array}{l}\text { Griffith } \\
\text { et al. } 2019 \\
\text { [44] }\end{array}$ & $\begin{array}{l}\text { Determination how different disease } \\
\text { frameworks impact the prevalence of } \\
\text { multimorbidity and its association with } \\
\text { patient-important outcomes }\end{array}$ & $\begin{array}{l}\text { Baseline } \\
\text { analysis of a } \\
\text { population- } \\
\text { based cohort } \\
\text { study }\end{array}$ & $\begin{array}{l}2 \text { terms: } \\
\text { - patient-important } \\
\text { functional outcome } \\
\text { - patient-important } \\
\text { outcome }\end{array}$ & $\begin{array}{l}\text { Generic; } \\
\text { community- } \\
\text { living adults } \\
\text { aged } 45 \text { to } 85 \\
\text { years }\end{array}$ & $\begin{array}{l}\text { - functional disability [OARS } \\
\text { questionnaire] } \\
\text { - social participation restriction } \\
\text { - self-rated physical health } \\
\text { - self-rated mental health }\end{array}$ \\
\hline $\begin{array}{l}\text { Nilsdotter } \\
\text { et al. } 2003 \\
\text { [45] }\end{array}$ & $\begin{array}{l}\text { Evaluation of long-term patient- } \\
\text { relevant outcomes after unilateral total } \\
\text { hip replacement for osteoarthritis }\end{array}$ & $\begin{array}{l}\text { Case-control- } \\
\text { study }\end{array}$ & $\begin{array}{l}1 \text { term: } \\
\text { - patient relevant } \\
\text { outcome }\end{array}$ & $\begin{array}{l}\text { Unilateral total } \\
\text { hip } \\
\text { replacement } \\
\text { for } \\
\text { osteoarthritis }\end{array}$ & $\begin{array}{l}\text { - general health status [SF-36] } \\
\text { - knee-specific health status: pain, } \\
\text { stiffness, physical function } \\
\text { [WOMAC] } \\
\text { - postoperative complications } \\
\text { - general comorbidity } \\
\text { - musculoskeletal comorbidity: need } \\
\text { of walking assistance, walking } \\
\text { distance, pain, need for analgesics, } \\
\text { joint replacement in contralateral } \\
\text { hip or in knee, fractures } \\
\text { - patient satisfaction }\end{array}$ \\
\hline $\begin{array}{l}\text { Paradowski } \\
\text { et al. } 2004 \\
\text { [46] }\end{array}$ & $\begin{array}{l}\text { Assessment of variation in knee pain, } \\
\text { function, and quality of life over two } \\
\text { years after removal of knee meniscus in } \\
\text { patients with and and without } \\
\text { radiographic knee osteoarthritis }\end{array}$ & $\begin{array}{l}\text { Prospective } \\
\text { follow-up } \\
\text { study }\end{array}$ & $\begin{array}{l}1 \text { term: } \\
\text { - patient-relevant } \\
\text { outcome }\end{array}$ & Meniscectomy & $\begin{array}{l}\text { - knee-specific health status: pain, } \\
\text { symptoms, activities of daily life, } \\
\text { sports and recreation function, } \\
\text { knee-related quality of life [KOOS] }\end{array}$ \\
\hline $\begin{array}{l}\text { Porat et al. } \\
2004[47]\end{array}$ & $\begin{array}{l}\text { Identification of the consequences of } \\
\text { an anterior cruciate ligament tear } 14 \\
\text { years after injury in a cohort of male } \\
\text { soccer players regarding radiographic } \\
\text { knee osteoarthritis } \\
\text { and patient-relevant outcomes }\end{array}$ & $\begin{array}{l}\text { Prospective } \\
\text { cohort study }\end{array}$ & $\begin{array}{l}1 \text { term: } \\
\text { - patient relevant } \\
\text { outcome }\end{array}$ & $\begin{array}{l}\text { Anterior } \\
\text { cruciate } \\
\text { ligament tear }\end{array}$ & $\begin{array}{l}\text { - general health status [SF-36] } \\
\text { - knee-specific health status: pain, } \\
\text { symptoms, activities of daily life, } \\
\text { sports and recreation function, } \\
\text { knee-related quality of life, func- } \\
\text { tional disability [KOOS, Lysholm } \\
\text { Knee Score] }\end{array}$ \\
\hline $\begin{array}{l}\text { Ramar et al. } \\
2017 \text { [48] }\end{array}$ & $\begin{array}{l}\text { Synthesis of the literature with regard } \\
\text { to models of care targeting patient- }\end{array}$ & $\begin{array}{l}\text { Systematic } \\
\text { review \& }\end{array}$ & $\begin{array}{l}2 \text { terms: } \\
\text { - patient-important }\end{array}$ & $\begin{array}{l}\text { Maintenance } \\
\text { dialysis care }\end{array}$ & $\begin{array}{l}\text { - mortality } \\
\text { - hospitalization }\end{array}$ \\
\hline
\end{tabular}


Table 4 Characteristics of the 13 studies not providing any justification for the patient relevance of outcomes (Continued)

\begin{tabular}{|c|c|c|c|c|c|}
\hline Authors & Objective/ motivation of article & $\begin{array}{l}\text { Type of } \\
\text { article/ study }\end{array}$ & $\begin{array}{l}\text { Terms used } \\
\text { synonymously }\end{array}$ & $\begin{array}{l}\text { Indicator } \\
\text { disease/ } \\
\text { intervention }\end{array}$ & $\begin{array}{l}\text { Outcomes considered patient- } \\
\text { relevant [instrument, if applicable] }\end{array}$ \\
\hline & $\begin{array}{l}\text { important outcomes for maintenance } \\
\text { dialysis patients focusing on } \\
\text { hospitalization and mortality }\end{array}$ & meta-analysis & $\begin{array}{l}\text { outcome } \\
\text { - patient outcome }\end{array}$ & & \\
\hline $\begin{array}{l}\text { Schnabel } \\
\text { et al. } 2014 \\
\text { [49] }\end{array}$ & $\begin{array}{l}\text { Comparison of the analgesic efficacy } \\
\text { and safety of ultrasound and nerve } \\
\text { stimulator guided peripheral nerve } \\
\text { catheters for postoperative pain } \\
\text { therapy }\end{array}$ & $\begin{array}{l}\text { Retrospective } \\
\text { database } \\
\text { analysis }\end{array}$ & $\begin{array}{l}4 \text { terms: } \\
\text { - patient-relevant tar- } \\
\text { get parameter } \\
\text { - patient-related } \\
\text { outcome } \\
\text { - patient-relevant par- } \\
\text { ameter [German: } \\
\text { Patienten-relevanter } \\
\text { Parameter] } \\
\text { - patient-relevant effi- } \\
\text { cacy parameter [Ger- } \\
\text { man: Patienten- } \\
\text { relevanter } \\
\text { Effektivitätsparameter] }\end{array}$ & $\begin{array}{l}\text { Peripheral } \\
\text { nerve } \\
\text { catheters for } \\
\text { pain therapy }\end{array}$ & $\begin{array}{l}\text { - postoperative pain } \\
\text { - postoperative need for additional } \\
\text { opioids } \\
\text { - cumulative local anesthetic } \\
\text { consumption } \\
\text { - puncture-associated complications } \\
\text { - postoperative catheter-related } \\
\text { complications }\end{array}$ \\
\hline $\begin{array}{l}\text { Stallmach } \\
\text { et al. } 2015 \\
\text { [50] }\end{array}$ & $\begin{array}{l}\text { Examination of possible improvements } \\
\text { in the clinical situation of patients with } \\
\text { inflammatory bowel disease in } \\
\text { Germany, focusing on patient-relevant } \\
\text { endpoints }\end{array}$ & $\begin{array}{l}\text { Secondary } \\
\text { data analysis }\end{array}$ & $\begin{array}{l}1 \text { term: } \\
\text { - patient-relevant } \\
\text { endpoint }\end{array}$ & $\begin{array}{l}\text { Inflammatory } \\
\text { bowel diseases }\end{array}$ & $\begin{array}{l}\text { - number of stationary treatments } \\
\text { per year (cases) } \\
\text { - average residence time } \\
\text { - number of operations } \\
\text { - inability to work } \\
\text { - premature mortality }\end{array}$ \\
\hline $\begin{array}{l}\text { W-Dahl } \\
\text { et al. } 2005 \\
\text { [51] }\end{array}$ & $\begin{array}{l}\text { Evaluation of the patient-relevant out- } \\
\text { comes pain, function, and quality of life } \\
\text { during two years in patients operated } \\
\text { on for knee osteoarthritis with tibial } \\
\text { osteotomy }\end{array}$ & $\begin{array}{l}\text { Prospective } \\
\text { follow-up } \\
\text { study }\end{array}$ & $\begin{array}{l}1 \text { term: } \\
\text { - patient-relevant } \\
\text { outcome }\end{array}$ & $\begin{array}{l}\text { Tibial } \\
\text { osteotomy for } \\
\text { uni- } \\
\text { compartmental } \\
\text { knee } \\
\text { osteoarthritis }\end{array}$ & $\begin{array}{l}\text { - knee-specific health status: pain, } \\
\text { symptoms, activities of daily life, } \\
\text { sports and recreation function, } \\
\text { knee-related quality of life [KOOS] } \\
\text { - complications, e.g., delayed } \\
\text { healing, deep venous thrombosis }\end{array}$ \\
\hline
\end{tabular}

articles $[4,5,27-34]$ or did not even provide a justification as to why the reported outcomes were considered to be relevant to patients $[3,40-51]$. With respect to both of these justifications, Murad et al. (2011) pointed out that neither outcomes considered patient-relevant in other studies nor those described as relevant to patients from a researchers' perspective necessarily represent what really matters to patients [20]. In addition, our subgroup analysis on studies involving patients and/or experts suggested that outcomes defined solely by experts in sense of health care professionals might not necessarily represent the patients' perspectives as well. Transferring this to practice implies that patient-driven approaches are required: Patients or representatives need to be asked when defining what is relevant for them. Referring to the patients' or - in the case of young children or critical care - the caregivers' opinions, such patientdriven approaches were identified in 14 articles included in our scoping review, but were limited to specific diseases such as cardiovascular disorders [11-13, 15-25]. For these particular patients, adverse events like myocardial infarction or stroke might be important outcomes but may not necessarily be applicable to other patient groups as specific diseases are commonly associated with very specific needs and preferences that cannot be generalized.

The number of outcomes identified in the context of patient relevance with 32 inductive categories certainly exceeded our expectations. However, outcomes representing social dimensions including the ability to perform daily activities or to fulfil social functions were apparently underrepresented, especially in studies not involving patients and experts. The variety of outcome measures as well as the lack of a consistent concept with regard to outcomes representing patient relevance limit the comparability of study results and make it difficult to determine the particular patient benefit of different interventions. This problem is also known from patientcentered care: Based on a systematic review and concept analysis of 417 articles, Scholl et al. (2014) found that models on patient-centeredness lack conceptual clarity, resulting in heterogeneous terminology and inconsistent outcome measures [52]. Even in the context of patientrelevant outcomes, this criticism was already voiced years ago, for example by Cleveringa et al. (2010) [42].

Meanwhile, promising initiatives like the European Qualitative research project on Patient-preferred outcomes in Early Rheumatoid Arthritis [53] or the 
Table 5 Terms used for supposedly patient-relevant outcomes ( $n=44$ articles)

\begin{tabular}{|c|c|}
\hline $\begin{array}{l}\text { Term, identified in the } \\
\text { (international) literature }\end{array}$ & $\begin{array}{l}\text { Number of articles } \\
\text { using this term (\%) }\end{array}$ \\
\hline Patient-important outcome & $23(52.3)$ \\
\hline Patient-relevant outcome & $17(38.6)$ \\
\hline Patient-relevant benefit & $4(9.1)$ \\
\hline Patient preference & $3(6.8)$ \\
\hline Patient outcome & $3(6.8)$ \\
\hline Patient-relevant endpoint & $2(4.5)$ \\
\hline Patient-centred outcome & $2(4.5)$ \\
\hline Patient-reported outcome & $2(4.5)$ \\
\hline Patient-relevant treatment benefit & $2(4.5)$ \\
\hline Patient endpoint & $1(2.3)$ \\
\hline Patient-prioritised outcome & $1(2.3)$ \\
\hline Patient-preferred outcome & $1(2.3)$ \\
\hline Patient-preferred health and treatment outcome & $1(2.3)$ \\
\hline Patient-reported benefit & $1(2.3)$ \\
\hline Patient-relevant health outcome & $1(2.3)$ \\
\hline Patient-oriented outcome & $1(2.3)$ \\
\hline Patient-relevant clinical trial outcome & $1(2.3)$ \\
\hline Self-reported outcome & $1(2.3)$ \\
\hline Patient-relevant measure & $1(2.3)$ \\
\hline Self-administered outcome measure & $1(2.3)$ \\
\hline Patient-relevant target parameter & $1(2.3)$ \\
\hline Patient-relevant parameter & $1(2.3)$ \\
\hline Patient-relevant efficacy parameter & $1(2.3)$ \\
\hline $\begin{array}{l}\text { Patient-important activity and participation } \\
\text { outcome }\end{array}$ & $1(2.3)$ \\
\hline Patient-important functional outcome & $1(2.3)$ \\
\hline Patient-important clinical outcome & $1(2.3)$ \\
\hline Child and family-important outcome & $1(2.3)$ \\
\hline Patient- and caregiver-relevant outcome & $1(2.3)$ \\
\hline Patient- and caregiver-relevant endpoint & $1(2.3)$ \\
\hline Patient-related outcome & $1(2.3)$ \\
\hline Patient-centered endpoint & $1(2.3)$ \\
\hline Patients' important outcome & $1(2.3)$ \\
\hline Patients' important treatment outcome & $1(2.3)$ \\
\hline Patient priority outcome & $1(2.3)$ \\
\hline Patient priority treatment outcome & $1(2.3)$ \\
\hline
\end{tabular}

International Consortium for Health Outcomes Measurement (ICHOM) [54] address this problem by defining core sets of outcomes that matter to patients. With a stronger focus on the operationalization of patientrelevant outcomes, a working group at the German Center for Health Services Research in Dermatology developed and validated a questionnaire assessing patient-relevant treatment benefits. This so-called Patient Benefit Index is available for different diseases, e.g. for the treatment of pruritus, lymphedema, and lipedema $[11,12]$. While such initiatives usually focus on individual diseases, the ICHOM has also started to develop outcome sets across diseases (e.g., overall adult health, older persons, overall pediatric health) [55]. Providing validated instruments for each outcome set, the ICHOM may have the potential to standardize outcome measurement in (clinical) trials, even though the initiative originally aimed to standardize the assessment of routine care data across countries to improve the quality of healthcare for common diseases worldwide.

Based on this scoping review we aimed to improve the conceptual clarity on patient relevance. Since the results do not allow us to derive a clear concept, in a next step we plan to close this gap by conducting a study among patients, health care professionals, and researchers. Unlike the approaches described above, we not only aim to identify a core set of generic rather than disease-specific patient-relevant outcomes, but also to contribute to a clear definition and understanding thereof. Based on the findings of this review, patients and health care professionals will be involved to define outcomes that are relevant to patients across diseases and important components of an adequate definition. Researchers will additionally ensure that the concept is feasible for research purposes. Our overarching long-term objective is to increase the comparability of study results with regard to patient relevance.

\section{Limitations}

A key strength of this scoping review is the systematic approach: The terms and outcomes were systematically and independently extracted by two researchers. Also, the literature search was not limited to fixed term sequences, but considered combinations of terms, e.g. "patient-relevant" and "outcome" instead of "patient-relevant outcome". Due to this approach, articles with titles including a statement or question like "Are outcomes reported patient-relevant?" were also covered. Nevertheless, the search strategy focused only on the titles of articles. Thus, it cannot be excluded that we missed relevant articles. This is further aggravated by the fact that the terminology used in the literature lacks consistency. For example, we did not consider search terms like "patient-centered outcomes" as we aimed to focus on outcomes that are relevant to patients, but these terms might have been used as synonyms in some articles as well. Finally, the restriction to full-text availability might represent another limitation. However, the abstracts alone did not provide sufficient information regarding supposedly patient-relevant outcomes and outcome justification. 


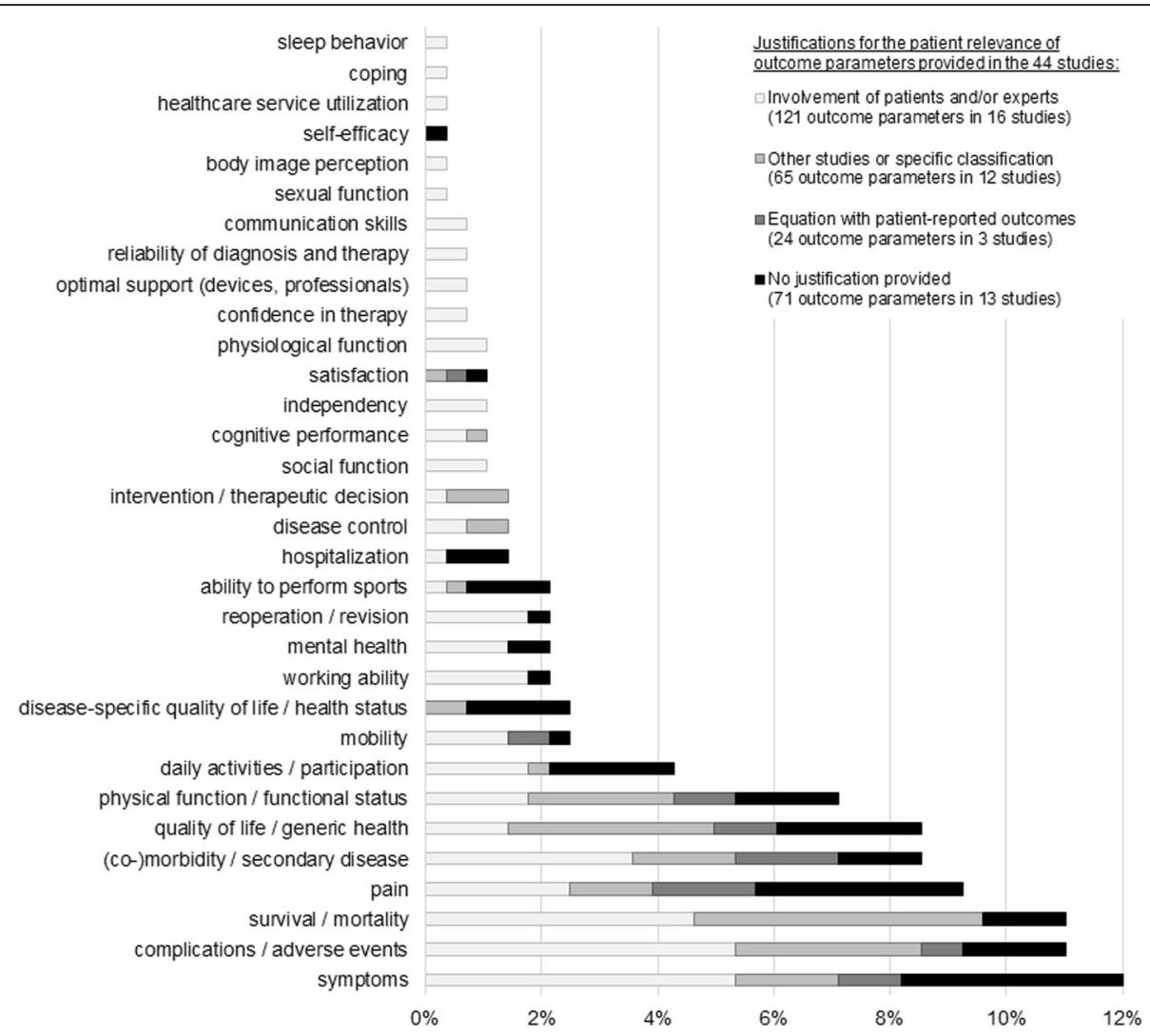

Fig. 2 Distribution of the 281 codes representing patient-relevant outcomes in 32 inductive categories (bars) with the distribution for each category grouped by the four justifications provided for the patient relevance of outcomes (shading); the percentages on the $x$-axis refer to 281

\section{Conclusions}

Returning to the initial question what outcomes are particularly relevant to patients and who determines their relevance, we conclude that recent studies use a variety of outcomes without asking patients what really matters to them. We were unable to identify either a sound definition or a consistent outcome set of patient-relevant outcomes, not even in the few studies that actively involved patients. In our opinion, consensus on a consistent terminology and set of generic patient-relevant outcomes is needed to adequately operationalize patient-centered care, increase the comparability of study results, and thereby enable patients to make choices regarding therapy in the context of shared decision-making.

Table 6 Summary of findings and implications for future research

\begin{tabular}{|c|c|c|}
\hline Research question & Findings & Implications for future research \\
\hline $\begin{array}{l}\text { What terminology is used for supposedly } \\
\text { patient-relevant outcomes? }\end{array}$ & $\begin{array}{l}\text { - large variety of terms found in the } \\
\text { international literature } \\
\text { - inconsistency of terms across and within } \\
\text { papers } \\
\text { - possible confusion between patient- } \\
\text { relevant and patient-reported }\end{array}$ & \multirow[t]{3}{*}{$\begin{array}{l}\text { - consistent concept of patient relevance is needed } \\
\text { - standard set of patient-relevant outcomes is needed } \\
\text { - patients must be involved when defining what is relevant } \\
\text { to them } \\
\text { - consistency regarding patient relevance will improve the } \\
\text { comparability of study results and enable patients to make } \\
\text { informed choices }\end{array}$} \\
\hline $\begin{array}{l}\text { What outcomes are considered to be } \\
\text { relevant to patients? }\end{array}$ & $\begin{array}{l}\text { - wide range of outcomes found } \\
\text { - commonly disease-specific outcomes } \\
\text { - social dimensions underrepresented }\end{array}$ & \\
\hline $\begin{array}{l}\text { What explanations are provided to justify } \\
\text { the relevance of these specific outcomes } \\
\text { for patients? }\end{array}$ & $\begin{array}{l}\text { - one third of studies without any } \\
\text { explanation } \\
\text { - another third refers to the opinion of } \\
\text { patients, experts, or caregivers as patient } \\
\text { representatives } \\
\text { - outcomes exclusively defined by experts } \\
\text { do not necessarily represent patients' } \\
\text { preferences }\end{array}$ & \\
\hline
\end{tabular}




\section{Supplementary information}

Supplementary information accompanies this paper at https://doi.org/1 0.1186/s12913-020-05442-9

Additional file 1. Full electronic search strategy for Embase, PubMed, Cochrane Central, Scopus, and Google Scholar.

\section{Abbreviations}

DES-SF: Diabetes Empowerment Scale-Short Form; DHP-18: Diabetes Health Profile (18 questions); DNVF: German Network for Health Services Research; DTSQ-status: Diabetes Treatment Satisfaction Questionnaire; EQ-5D: European Quality of Life questionnaire - 5 Dimensions; HADS: Hospital Anxiety and Depression Scale; IES-R: Impact of Event Scale-Revised; IQEHC: Institute for Quality and Efficiency in Health Care; KOOS: Knee Injury and Osteoarthritis Outcome Score; LOST-IT: LOST to follow-up Information in Trials; OARS questionnaire: Older American Resources and Services questionnaire; SF36: Short Form-36 Health Survey; WOMAC: Western Ontario and McMaster Universities Osteoarthritis Index

\section{Acknowledgements}

We thank Dorothea Kesztyüs, MPH, Institute of General Medicine, Ulm University, for conducting the literature search in Embase.

\section{Authors' contributions}

$A B$ and $C K$ developed the idea and concept for the study. CK conducted the literature search and prepared the search results for analysis. The literature was analyzed by CK and MK under the supervision of AB. CK drafted the first version of the manuscript, which was critically reviewed by $A B$. All authors read and approved the final manuscript.

\section{Funding}

Not applicable.

\section{Availability of data and materials}

All data generated or analyzed during this study are included in this published article.

\section{Ethics approval and consent to participate}

Not applicable.

\section{Consent for publication}

Not applicable.

\section{Competing interests}

The authors declare that they have no competing interests.

\section{Author details}

${ }^{1}$ Institute of General Practice and Interprofessional Care, Faculty of Health/ School of Medicine, Witten/Herdecke University, Alfred-Herrhausen-Str. 50, 58448 Witten, Germany. ${ }^{2}$ Institute of General Medicine, Ulm University,

Albert-Einstein-Allee 23, 89081 Ulm, Germany.

Received: 6 February 2020 Accepted: 18 June 2020

Published online: 29 June 2020

\section{References}

1. American Geriatrics Society Expert Panel on Person-Centered Care. Personcentered care: a definition and essential elements. J Am Geriatr Soc. 2016; 64:15-8. https://doi.org/10.1111/jgs.13866.

2. Elwyn G, Frosch D, Thomson R, Joseph-Williams N, Lloyd A, Kinnersley P, et al. Shared decision making: a model for clinical practice. J Gen Intern Med. 2012;27:1361-7. https://doi.org/10.1007/s11606-012-2077-6.

3. Gandhi GY, Murad MH, Fujiyoshi A, Mullan RJ, Flynn DN, Elamin MB, et al. Patient-important outcomes in registered diabetes trials. JAMA. 2008;299: 2543-9. https://doi.org/10.1001/jama.299.21.2543

4. Adie S, Harris IA, Naylor JM, Mittal R. Are outcomes reported in surgical randomized trials patient-important? A systematic review and meta-analysis. Can J Surg. 2017;60:86-93.

5. Gaudry S, Messika J, Ricard J-D, Guillo S, Pasquet B, Dubief E, et al. Patientimportant outcomes in randomized controlled trials in critically ill patients: a systematic review. Ann Intensive Care. 2017;7:28. https://doi.org/10.1186/ s13613-017-0243-z .

6. Institut für Qualität und Wirtschaftlichkeit im Gesundheitswesen. Allgemeine Methoden. 2017. https://www.iqwig.de/download/Allgemeine-Methoden_ Version-5-0.pdf. Accessed 9 June 2020.

7. Biomarkers Definitions Working Group. Biomarkers and surrogate endpoints: preferred definitions and conceptual framework. Clin Pharmacol Ther. 2001; 69:89-95. https://doi.org/10.1067/mcp.2001.113989.

8. Geraedts M, Drösler SE, Döbler K, Eberlein-Gonska M, Heller G, Kuske S, et al. Memorandum III, part 3: quality of care and patient safety methods. Gesundheitswesen. 2017;79:e95-e124. https://doi.org/10.1055/s-0043-112431

9. Tricco AC, Lillie E, Zarin W, O'Brien KK, Colquhoun H, Levac D, et al. PRISMA extension for scoping reviews (PRISMA-SCR): checklist and explanation. Ann Intern Med. 2018;169:467-73. https://doi.org/10.7326/M18-0850

10. Mayring P. Qualitative content analysis: theoretical foundation, basic procedures and software solution. 2014. https://nbn-resolving.org/urn:nbn: de:0168-ssoar-395173. Accessed 9 June 2020.

11. Blome C, Augustin M, Siepmann D, Phan NQ, Rustenbach SJ, Stander S. Measuring patient-relevant benefits in pruritus treatment: development and validation of a specific outcomes tool. Br J Dermatol. 2009;161:1143-8. https://doi.org/10.1111/j.1365-2133.2009.09328.x

12. Blome C, Augustin M, Heyer K, Knofel J, Cornelsen H, Purwins S, Herberger K. Evaluation of patient-relevant outcomes of lymphedema and lipedema treatment: development and validation of a new benefit tool. Eur J Vasc Endovasc Surg. 2014;47:100-7. https://doi.org/10.1016/j.ejvs.2013.10.009

13. Cho Y, Sautenet B, Gutman T, Rangan G, Craig JC, Ong AC, et al. Identifying patient-important outcomes in polycystic kidney disease: an international nominal group technique study. Nephrology (Carlton). 2019. https://doi.org/ 10.1111/nep.13566

14. Daeter EJ, Timmermans MJC, Hirsch A, Lipsic E, Houterman S, van Veghel D, van Boven WJ. Defining and measuring a standard set of patient-relevant outcomes in coronary artery disease. Am J Cardiol. 2018;121:1477-88. https://doi.org/10.1016/j.amjcard.2018.02.037

15. Dinglas VD, Faraone LN, Needham DM. Understanding patient-important outcomes after critical illness: a synthesis of recent qualitative, empirical, and consensus-related studies. Curr Opin Crit Care. 2018;24:401-9. https:// doi.org/10.1097/MCC.0000000000000533

16. Eiring O, Nylenna M, Nytroen K. Patient-important outcomes in the longterm treatment of bipolar disorder: a mixed-methods approach investigating relative preferences and a proposed taxonomy. Patient. 2016;9: 91-102. https://doi.org/10.1007/s40271-015-0128-x

17. Kinter ET, Schmeding A, Rudolph I, dos Reis S, Bridges JFP. Identifying patient-relevant endpoints among individuals with schizophrenia: An application of patient-centered health technology assessment. Int J Technol Assess Health Care. 2009;25:35-41. https://doi.org/10.1017/ S0266462309090059

18. Konkle BA, Skinner M, lorio A. Hemophilia trials in the twenty-first century: defining patient important outcomes. Res Pract Thromb Haemost. 2019;3: 184-92. https://doi.org/10.1002/rth2.12195

19. Lindsley KB, Hutfless S, Hawkins BS, Blim JF, Roberts D, Olsen TW, et al. Evaluation of clinical questions and patient-important outcomes associated with the treatment of age-related macular degeneration. JAMA Ophthalmol. 2018;136:1217-25. https://doi.org/10.1001/jamaophthalmol.2018.3456

20. Murad MH, Shah ND, van Houten HK, Ziegenfuss JY, Deming JR, Beebe TJ, et al. Individuals with diabetes preferred that future trials use patientimportant outcomes and provide pragmatic inferences. J Clin Epidemiol. 2011;64:743-8. https://doi.org/10.1016/j.jclinepi.2010.08.005

21. Nabbout R, Auvin S, Chiron C, Irwin J, Mistry A, Bonner N, et al. Development and content validation of a preliminary core set of patientand caregiver-relevant outcomes for inclusion in a potential composite endpoint for Dravet syndrome. Epilepsy Behav. 2018;78:232-42. https://doi. org/10.1016/j.yebeh.2017.08.029.

22. Sanderson T, Morris $M$, Calnan M, Richards $P$, Hewlett S. What outcomes from pharmacologic treatments are important to people with rheumatoid arthritis? Creating the basis of a patient core set. Arthritis Care Res (Hoboken). 2010;62:640-6. https://doi.org/10.1002/acr.20034

23. Sung W, Rogers RG, Barber MD, Clark MA. Conceptual framework for patient-important treatment outcomes for pelvic organ prolapse. Neurourol Urodyn. 2014;33:414-9. https://doi.org/10.1002/nau.22397.

24. Van der Elst K, Meyfroidt S, De Cock D, De Groef A, Binnard E, Moons P, et al. Unraveling patient-preferred health and treatment outcomes in early 
rheumatoid arthritis: a longitudinal qualitative study. Arthritis Care Res (Hoboken). 2016;68:1278-87. https://doi.org/10.1002/acr.22824 .

25. Wilson HA, Middleton R, Abram SGF, Smith S, Alvand A, Jackson WF, et al. Patient relevant outcomes of unicompartmental versus total knee replacement: systematic review and meta-analysis. BMJ. 2019;364:1352. https://doi.org/10.1136/bmj.l352 .

26. van Veghel D, Marteijn $M$, de Mol B. First results of a national initiative to enable quality improvement of cardiovascular care by transparently reporting on patient-relevant outcomes. Eur J Cardiothorac Surg. 2016;49: 1660-9. https://doi.org/10.1093/ejcts/ezw034

27. Ameur H, Ravaud P, Fayard F, Riveros C, Dechartres A. Systematic reviews of therapeutic interventions frequently consider patientimportant outcomes. J Clin Epidemiol. 2017;84:70-7. https://doi.org/10. 1016/j.jclinepi.2017.01.006

28. Fei Y, Guyatt GH, Alexander PE, El Dib R, Siemieniuk RAC, Vandvik PO, et al. Addition of Ezetimibe to statins for patients at high cardiovascular risk: systematic review of patient-important outcomes. J Eval Clin Pract. 2018;1: 222-31. https://doi.org/10.1111/jep.12663

29. Kvitkina T, ten Haaf A, Reken S, McGauran N, Wieseler B. Patient-relevant outcomes and surrogates in the early benefit assessment of drugs: first experiences. Zeitschrift für Evidenz Fortbildung und Qualität im Gesundheitswesen. 2014;108:528-38. https://doi.org/10.1016/j.zefq.2014.06.015

30. Roos EM, Roos HP, Ryd L, Lohmander LS. Substantial disability 3 months after arthroscopic partial meniscectomy: a prospective study of patientrelevant outcomes. Arthroscopy. 2000;16:619-26. https://doi.org/10.1053/ jars.2000.4818

31. Schumacher SG, Sohn H, Qin ZZ, Gore G, Davis JL, Denkinger CM, Pai M Impact of molecular diagnostics for tuberculosis on patient-important outcomes: a systematic review of study methodologies. PLoS One. 2016;11: e0151073. https://doi.org/10.1371/journal.pone.0151073 .

32. Singh M, Mealing S, Baculea S, Cote S, Whelan J. Impact of novel agents on patient-relevant outcomes in patients with previously untreated chronic lymphocytic leukemia who are not eligible for fludarabine-based therapy. J Med Econ. 2017;20:1066-73. https://doi.org/10.1080/13696998.2017.1357563.

33. Wieseler B, Wolfram N, McGauran N, Kerekes MF, Vervolgyi V, Kohlepp P, et al. Completeness of reporting of patient-relevant clinical trial outcomes: comparison of unpublished clinical study reports with publicly availablel data. PLoS Med. 2013;10:e1001526. https://doi.org/10.1371/journal.pmed. 1001526

34. Yordanov Y, Dechartres A, Ravaud P. Patient-important outcomes in systematic reviews: poor quality of evidence. PLoS One. 2018;13:e0195460. https://doi.org/10.1371/journal.pone.0195460

35. El Dib R, Tikkinen KAO, Akl EA, Gomaa HA, Mustafa RA, Agarwal A, et al. Systematic survey of randomized trials evaluating the impact of alternative diagnostic strategies on patient-important outcomes. J Clin Epidemiol. 2017; 84:61-9. https://doi.org/10.1016/j.jclinepi.2016.12.009

36. Fayed N, de Camargo OK, Elahi I, Dubey A, Fernandes RM, Houtrow A, Cohen E. Patient-important activity and participation outcomes in clinical trials involving children with chronic conditions. Qual Life Res. 2014;23:7517. https://doi.org/10.1007/s11136-013-0483-9 .

37. Nilsdotter A-K, Toksvig-Larsen S, Roos EM. A 5 year prospective study of patient-relevant outcomes after total knee replacement. Osteoarthr Cartil. 2009;17:601-6. https://doi.org/10.1016/j.joca.2008.11.007

38. Nilsdotter A-K, Isaksson F. Patient relevant outcome 7 years after total hip replacement for OA - a prospective study. BMC Musculoskelet Disord. 2010; 11:47. https://doi.org/10.1186/1471-2474-11-47

39. Nilsdotter AK, Lohmander LS. Patient relevant outcomes after total hip replacement. A comparison between different surgical techniques. Health Qual Life Outcomes. 2003;1:21. https://doi.org/10.1186/1477-7525-1-21

40. Agarwal A, Johnston BC, Vernooij RWM, Carrasco-Labra A, BrignardelloPetersen R, Neumann I, et al. Authors seldom report the most patientimportant outcomes and absolute effect measures in systematic review abstracts. J Clin Epidemiol. 2017:3-12. https://doi.org/10.1016/j.jclinepi.2016. 08.004

41. Cao H, Zhai J, Mu W, Lei X, Cao H, Liu C, Shang H. Use of comparative effectiveness research for similar Chinese patent medicine for angina pectoris of coronary heart disease: a new approach based on patient-important outcomes. Trials. 2014;15:84. https://doi.org/10.1186/1745-6215-15-84 .

42. Cleveringa FGW, Minkman MH, Gorter KJ, van den Donk M, Rutten GE. Diabetes care protocol: effects on patient-important outcomes. A cluster randomized, non-inferiority trial in primary care. Diabet Med. 2010;27:44250. https://doi.org/10.1111/j.1464-5491.2010.02968.x

43. Englund M, Roos EM, Roos HP, Lohmander LS. Patient-relevant outcomes fourteen years after meniscectomy: influence of type of meniscal tear and size of resection. Rheumatology (Oxford). 2001;40:631-9.

44. Griffith LE, Gilsing A, Mangin D, Patterson C, van den Heuvel E, Sohel N, et al. Multimorbidity frameworks impact prevalence and relationships with patient-important outcomes. J Am Geriatr Soc. 2019. https://doi.org/10. 1111/jgs.15921

45. Nilsdotter A-K, Petersson IF, Roos EM, Lohmander LS. Predictors of patient relevant outcome after total hip replacement for osteoarthritis: a prospective study. Ann Rheum Dis. 2003;62:923-30.

46. Paradowski PT, Englund M, Roos EM, Lohmander LS. Similar group mean scores, but large individual variations, in patient-relevant outcomes over 2 years in meniscectomized subjects with and without radiographic knee osteoarthritis. Health Qual Life Outcomes. 2004;2:38. https://doi.org/10.1186/ 1477-7525-2-38

47. von Porat A, Roos EM, Roos H. High prevalence of osteoarthritis 14 years after an anterior cruciate ligament tear in male soccer players: a study of radiographic and patient relevant outcomes. Ann Rheum Dis. 2004;63:269-73.

48. Ramar P, Ahmed AT, Wang Z, Chawla SS, Suarez MLG, Hickson L, et al. Effects of different models of Dialysis care on patient-important outcomes: a systematic review and meta-analysis. Popul Health Manag. 2017;6:495-505. https://doi.org/10.1089/pop.2016.0157.

49. Schnabel A, Middendorf B, Boschin MG, Gottschalk A, van Aken H, Zahn PK, Pogatzki-Zahn EM. Differences of analgesic efficacy and complication rates between ultrasound and nervestimulator guided peripheral nerve catheters: database analysis on patient-relevant target parameters. Der Anästhesist. 2014;63:825-31. https://doi.org/10.1007/s00101-014-2379-7

50. Stallmach A, Dennler U, Marschall U, Schmidt C. Patient-relevant endpoints in inflammatory bowel diseases - have changes occurred in Germany over the past twelve years? J Crohns Colitis. 2015;9:390-7. https://doi.org/10. 1093/ecco-jcc/jjv041.

51. W-Dahl A, Toksvig-Larsen S, Roos EM. A 2-year prospective study of patientrelevant outcomes in patients operated on for knee osteoarthritis with tibial osteotomy. BMC Musculoskelet Disord. 2005;6:18. https://doi.org/10.1186/ 1471-2474-6-18

52. Scholl I, Zill JM, Harter M, Dirmaier J. An integrative model of patientcenteredness - a systematic review and concept analysis. PLoS One. 2014;9: e107828. https://doi.org/10.1371/journal.pone.0107828.

53. van der Elst K, Bremander A, de Groef A, Larsson I, Mathijssen EGE, Vriezekolk JE, et al. European qualitative research project on patientpreferred outcomes in early rheumatoid arthritis (EQPERA): rationale, design and methods of a multinational, multicentre, multilingual, longitudinal qualitative study. BMJ Open. 2019;9:e023606. https://doi.org/10.1136/ bmjopen-2018-023606 .

54. International Consortium for Health Outcome Measurement. ICHOM. https://www.ichom.org/. Accessed 4 June 2020.

55. International Consortium for Health Outcome Measurement. ICHOM Standard Sets. https://www.ichom.org/standard-sets/. Accessed 4 June 2020.

\section{Publisher's Note}

Springer Nature remains neutral with regard to jurisdictional claims in published maps and institutional affiliations.

\section{Ready to submit your research? Choose BMC and benefit from:}

- fast, convenient online submission

- thorough peer review by experienced researchers in your field

- rapid publication on acceptance

- support for research data, including large and complex data types

- gold Open Access which fosters wider collaboration and increased citations

- maximum visibility for your research: over $100 \mathrm{M}$ website views per year

At $\mathrm{BMC}$, research is always in progress.

Learn more biomedcentral.com/submissions 